\title{
Optimizing Toll Locations and Levels Using a Mixed Integer Linear Approximation Approach
}

\author{
Joakim Ekström, Agachai Sumalee and Hong K. Lo
}

\section{Linköping University Post Print}

\section{Tweet}

N.B.: When citing this work, cite the original article.

Original Publication:

Joakim Ekström, Agachai Sumalee and Hong K. Lo, Optimizing Toll Locations and Levels Using a Mixed Integer Linear Approximation Approach, 2012, Transportation Research Part B: Methodological, (46), 7, 834-854.

http://dx.doi.org/10.1016/j.trb.2012.02.006

Copyright: Elsevier

http://www.elsevier.com/

Postprint available at: Linköping University Electronic Press

http://urn.kb.se/resolve?urn=urn:nbn:se:liu:diva-76641 


\title{
Optimizing toll locations and levels using a mixed integer linear approximation approach
}

\author{
Joakim Ekström ${ }^{\mathrm{a}, *}$, Agachai Sumalee ${ }^{\mathrm{b}}$, Hong K. Lo ${ }^{\mathrm{c}}$ \\ ${ }^{a}$ Department of Science and Technology, Linköping University, SE-601 74 Norrköping, \\ Sweden \\ ${ }^{b}$ Department of Civil and Structural Engineering, Hong Kong Polytechnic University, \\ Kowloon, Hong Kong \\ ${ }^{c}$ Department of Civil Engineering, Hong Kong University of Science and Technology, Clear \\ Water Bay, Hong Kong
}

\begin{abstract}
This paper addresses the toll design problem of finding the toll locations and levels in a congestion pricing scheme, which minimize the total travel time and the toll-point cost (set-up and operational costs of the toll collecting facilities). Road users in the network are assumed to be distributed according to the principle of user equilibrium, with the demand assumed to be fixed and given a priori. The toll design problem is commonly formulated as a non-linear program, which in general is non-convex and non-smooth, and thus difficult to solve for a global optimum. In this paper, the toll design problem is approximated by a mixed integer linear program (MILP), which can be solved to its globally optimal solution. The MILP also gives a lower bound estimation of the original non-linear problem, and the accuracy of the approximation is improved by iteratively updating the MILP. To demonstrate the approach, we apply the algorithm to two networks: a smaller network with 18 links and 4 OD-pairs to illustrate the properties of the approach, and the Sioux Falls network with 87 links and 30 OD-pairs to demonstrate the applicability of the approach.
\end{abstract}

Keywords: congestion pricing, network design, global optimization, bi-level optimization

\footnotetext{
*Corresponding author

Email addresses: joaek@itn.liu.se (Joakim Ekström ), ceasumal@polyu.edu.hk (Agachai Sumalee), cehklo@ust.hk (Hong K. Lo)
} 


\section{Introduction}

When congestion pricing is implemented in practice, two questions arise, namely where to collect the tolls, and what toll levels to charge. The application of marginal social cost pricing (MSCP), first introduced by Pigou (1920) and Knight (1924), leads to the most efficient usage of the transportation network, minimizing the total travel time (if the demand is fixed) or maximizing the social surplus (if the demand is elastic). There are other pricing schemes which give the same result as the MSCP scheme, and usually all such schemes are denoted as first-best pricing schemes. If restrictions are imposed on the toll locations and/or levels, or if the cost of deploying the toll collecting facilities is included in the objective, there is no guarantee that the pricing scheme which maximizes the social surplus is the first-best one. In fact, if there is a high enough cost associated with deploying the toll collecting facilities or if the possible toll locations are restricted, it is likely that the pricing scheme which maximizes the social surplus may not correspond to the first-best one and such pricing schemes are therefore considered as second-best.

The most common version of the second-best problem is the toll level setting problem (TLP) in which the toll locations are predetermined. Marchand (1968), Verhoef et al. (1996), Liu and McDonald (1999) and Yin and Lawphongpanich (2009) investigate the properties of the second-best optimal toll level solution, and Verhoef (2002b), Lawphongpanich and Hearn (2004) and Shepherd and Sumalee (2004) develop methods for finding second-best optimal toll levels in large networks. The toll design problem (TDP) instead involves both decision of toll locations and their corresponding toll levels. Verhoef (2002a) introduce the problem of optimal selection of toll points, and the problem is further discussed in May et al. (2002) and Shepherd and Sumalee (2004). The problem is further constrained by Yang and Zhang (2003), Sumalee (2004) and Zhang and Yang (2004) to only allow toll locations which form cordons.

Both the continuous TLP and the discrete TDP can be considered as special cases of the network design problem (NDP), which was first introduced by Leblanc (1975) for capacity enhancements of road infrastructure. The NDP is generally non-convex, and has intrigued transportation economist, traffic engineers and mathematicians for over three decades. For a comprehensive review of the NDP, see Yang and Bell (1998). In recent years, various methods have been suggested for solving different versions of the NDP. Meng and Yang (2002) formulates a NDP for continuous road capacity enhancements which is solved with a method based on simulated annealing, and Gao et al. (2005) applies an algorithm based on generalized benders decomposition to solve the NDP for the case of adding road infrastructure. Recently, Wang and Lo (2010) suggested a mixed integer linear program (MILP) approximation of the NDP, for continuous capacity enhancements, which can be solved to its globally optimal solution.

In the TDP, we search for second-best optimal toll locations and their corresponding toll levels, leading to a non-linear program with both continuous (toll 
levels) and discrete (toll locations) variables. Hearn and Ramana (1998) present and solve the problem of finding the first-best pricing scheme which uses the least number of toll facilities. If the cost of setting up and operating the toll collection system is considered, a first-best pricing scheme may not be an optimal one, even if it is the one with the least number of toll facilities. Applying the method by Hearn and Ramana however gives an important bound on the number of toll points required to reach the first-best solution. To solve the toll location problem, which in general is non-convex, Verhoef (2002a) suggests a heuristic procedure based on a location index computable for each link. This method may, however, miss to recognize toll locations which yield a high benefit only if they are tolled simultaneously. In Yang and Zhang (2003) and Shepherd and Sumalee (2004), genetic algorithms are developed to solve the problem, and in Ekström et al. (2009) a smoothing technique is applied to approximate the discrete variables by a continuous function. None of these methods address the global optimality of the TDP, and no tight lower bound estimation, to which the solution can be compared, exists.

In this paper the global optimality of the TDP is addressed by formulating a MILP approximation, which can be solved to its globally optimal solution. The general outline of the MILP approximation follows Wang and Lo (2010), while the actual MILP formulation for the TDP takes advantage of problem specific features for a more efficient MILP formulation. In contrast to the NDP, the functions related to travel time are only dependent on the link flow, and the toll revenue function, which is the only function which includes both link flow and toll level, will always take the form of a bilinear term. Also, the formulation of the MILP approximation presented in this paper is guaranteed to give an optimistic or lower bound estimation of the TDP.

Assuming that the road users are distributed according to the principle of deterministic user equilibrium (UE) in the network, both the NDP and TDP can be expressed as bi-level programs. In the bi-level program the overall objective is given by the upper level program and the UE conditions are represented on the lower level as a convex program. The UE can also be formulated as a variational inequality (VI), and by applying the VI as constraints to the NDP or TDP, a single level formulation can be obtained. The MILP approximation of the NDP in Wang and Lo (2010) relies on a path based formulation of the VIconstraints. Our MILP approximation of the TDP is instead link-based, which obviates the need of path enumeration, thereby enabling it for solving larger network problems.

To reduce the error associated with the approximation of the total travel time, travel time, and link toll revenue functions, the link flow solution from the MILP will be used to iteratively improve the approximation. Each such iteration will move the optimal objective function value of the MILP towards the global optimum of the toll design problem.

The VI-constraints, used to represent the lower level problem, are defined based on all feasible link flows, but the feasible set of link flows makes up a polyhedral 
set, and the VI-constraints can therefore instead be defined based on a finite number of extreme points. The number of extreme points is still large, and to solve the MILP, a cutting constraint algorithm (CCA) is applied to generate one extreme point at a time. Marcotte (1983) applies a similar CCA algorithm to solve a NDP for continuous capacity enhancement and Lawphongpanich and Hearn (2004) used a CCA to solve the TLP when the set of tollable links is restricted. However, both these implementations of the CCA algorithm are performed on the non-convex TLP and can only give a local optimum.

To demonstrate the MILP approximation approach, we apply the MILP-CCA on two networks: a smaller network with 18 links and 4 OD pairs to illustrate the properties of the approach, and the Sioux Falls network with 87 links and 30 OD pairs to demonstrate the applicability of the approach. The Sioux Falls network is more or less the largest problem tested in the literature, when solving the toll location problem to its global optimum.

The remainder of this paper is outlined as follows. In Section 2, the TDP is presented and in Section 3 the MILP approximation is presented. The solution algorithm is described in Section 4, and the numerical results follow in Section 5. In Section 6, we provide some concluding remarks and suggest further research directions.

\section{Problem formulation}

\subsection{The traffic model}

The traffic network is modeled by a set of nodes $\mathcal{N}$, and a set of links $\mathcal{A}$, each link connecting the node pair $\left\{n_{s}, n_{e}\right\} \in \mathcal{N}$, where $n_{s}$ and $n_{e}$ denote the start and end node respectively of the link. The set of OD pairs $\mathcal{I}$ makes up the set of node pairs for which the demand $q_{i}$ is specified. Let $v$ be the vector of link flows, with $v_{a}$ corresponding to the flow on link $a$. Furthermore, let $x^{i}$ be the vector of link flows induced by the demand in OD pair $i \in \mathcal{I}$. The set of feasible link flows can then be formulated as

$$
\Omega=\left\{v: v=\sum_{i \in I} x^{i}, A x^{i}=b_{i}, x^{i} \geq 0, i \in I\right\}
$$

where $A$ is the link-node incidence matrix and for each link there are two entries with the position $n_{s}$ equal to -1 and the position of $n_{e}$ equal to 1 . The vector $b_{i}$ has a length equal to the number of nodes, with the element at the position of the origin node equal to $-q_{i}$ and that of the destination node equal to $q_{i}$. The link travel time is assumed to be a strictly monotone increasing function $t_{a}$ of $v_{a}$. The cost of traveling on link $a$ is made up of both the link travel time and the link toll, $\tau_{a}$, and is expressed as $c_{a}\left(\tau_{a}, v_{a}\right)=\alpha t_{a}\left(v_{a}\right)+\tau_{a}$, where $\alpha$ is the value of time. 
The route choice model assumes that within an OD pair, road users choose a route with minimum cost in the traffic network, and no user can reduce their travel cost by changing route. This is referred to as Wardrop's user equilibrium (Wardrop, 1952) or a user optimal route choice. The output from the route choice model will be the link flows, $v$, corresponding to the toll vector $\tau$.

The equilibrium solution is obtained by solving (Sheffi, 1984):

$$
U E(\tau)=\min _{v \in \Omega} G(v)=\sum_{a \in \mathcal{A}} \int_{0}^{v_{a}} c_{a}\left(\tau_{a}, w\right) d w
$$

\subsection{The toll design problem (TDP)}

The TDP is formulated as a bi-level mathematical program, with the upper level formulation stated as

$$
\min _{(\tau, y) \in \Phi} F_{\mathrm{TDP}}(\tau, v(\tau), y)=\alpha \sum_{a \in \mathcal{A}} t_{a}\left(v_{a}(\tau)\right) v_{a}(\tau)+g_{a}\left(v_{a}(\tau), y_{a}\right),
$$

where $y_{a}$ is equal to 1 if link $a$ is tolled and 0 otherwise. The function $g_{a}\left(v_{a}(\tau), y_{a}\right)$ gives the setup and operational cost of locating a toll on link $a$, and can depend on the link flow to capture the transaction cost of each passing vehicle. The set

$$
\Phi=\left\{(\tau, y): \tau_{a} \leq y_{a} \tau_{a}^{U}, \tau_{a} \geq 0, y_{a}=\{0,1\}, a \in \mathcal{A}\right\}
$$

describes the feasible toll levels and locations, where $\tau_{a}^{U}$ is an upper bound on the toll level for link $a$, which can be relaxed by setting $\tau^{U}$ high enough. The lower level problem is the route choice model formulated in (2), and the link flows $v(\tau)$ are given implicitly by the solution to this problem. On the upper level, the aim is to minimize the sum of the total travel time in the network and the setup and operational costs of the toll facilities. The bi-level program is in general non-convex and non-smooth, and therefore difficult to solve for a global optimum.

Note that the route choice model can be expressed by the variational inequalities (VI) (Dafermos, 1980b)

$$
c(\tau, v)^{T}(v-\tilde{v}) \leq 0, \quad \tilde{v} \in \Omega
$$

The bi-level program can then be reformulated as a single level problem by expressing the lower level problem as a set of VI-constraints. The single level problem is:

$$
\begin{gathered}
\min _{(\tau, y) \in \Phi, v \in \Omega} F_{\mathrm{TDP}}(\tau, v, y)=\alpha \sum_{a \in A} t_{a}\left(v_{a}\right) v_{a}+\sum_{a \in A} g_{a}\left(v_{a}, y_{a}\right) \\
\text { subject to } \\
c(\tau, v)^{T}(v-\tilde{v}) \leq 0, \quad \tilde{v} \in \Omega .
\end{gathered}
$$


The VI is defined based on all feasible link flows, $\Omega$. By adding bounds on the link flows $v, \Omega$ can be treated as a bounded polyhedron, and these bounds can be relaxed by setting them high enough. Any feasible link flow $\tilde{v}$ can then be expressed as the convex combination of the extreme points of $\Omega$, i.e. $\tilde{v}=$ $\sum_{s=1}^{S} \lambda_{s} \hat{v}^{s}$, where $\hat{v}^{s}$ denotes the $s^{t h}$ extreme point of $\Omega(S$ is the finite number of extreme points), $\sum_{s=1}^{S} \lambda_{s}=1$ and $0 \leq \lambda_{s} \leq 1$. We can thus reformulate the VI-constraints as:

$$
c(\tau, v)^{T}\left(v-\hat{v}^{s}\right) \leq 0, \quad s \in 1 \ldots S .
$$

Lemma 2.1. Any feasible link flow $v$ satisfying $c(\tau, v)^{T}\left(v-\hat{v}^{s}\right) \leq 0, s \in 1 \ldots S$ is also feasible in $c(\tau, v)^{T}(v-\tilde{v}) \leq 0, \tilde{v} \in \Omega$.

Proof. Express $\tilde{v}$ by the extreme points of $\Omega, \tilde{v}=\sum_{s=1}^{S} \lambda_{s} \hat{v}^{s}$ with $0 \leq \lambda_{s} \leq 1$. Then the left hand side of (4) can be expressed as $c(\tau, v)^{T}\left(v-\sum_{s=1}^{S} \lambda_{s} \hat{v}^{s}\right)$, which can be rendered as $\sum_{s=1}^{S} \lambda_{s} c(\tau, v)^{T}\left(v-\hat{v}^{s}\right)$. Then any $v$ satisfying $c(\tau, v)^{T}(v-$ $\left.\hat{v}^{s}\right) \leq 0, \quad s \in 1 \ldots S$, clearly must also satisfy $\sum_{s=1}^{S} \lambda_{s} c(\tau, v)^{T}\left(v-\hat{v}^{s}\right) \leq 0$.

Based on Lemma 2.1 we can rewrite problem (5) as

$$
\begin{aligned}
& \min _{(\tau, y) \in \Phi, v \in \Omega} F_{\mathrm{TDP}}(\tau, v, y)=\alpha \sum_{a \in A} t_{a}\left(v_{a}\right) v_{a}+\sum_{a \in A} g_{a}\left(v_{a}, y_{a}\right) \\
& \text { subject to } \quad c(\tau, v)^{T}\left(v-\hat{v}^{s}\right) \leq 0, \quad s \in 1 \ldots S
\end{aligned}
$$

Proposition 2.1. If $v$ and $\tau$ are a solution to (7), then $v$ and $\tau$ also solves (5).

Proof. The only difference between (5) and (7) is the substitution of $c(\tau, v)^{T}(v-$ $\tilde{v}) \leq 0, \tilde{v} \in \Omega$ by $c(\tau, v)^{T}\left(v-\hat{v}^{s}\right) \leq 0, s \in 1 \ldots S$, and hence the proof follows directly from Lemma 2.1 .

\section{The TDP approximated by a mixed integer linear program}

The sets $\Phi$ and $\Omega$ in (7) only includes linear terms, and the only non-linear terms in (7) appear in the objective function and the VI-constraints. In this section the non-linear terms will therefore be approximated by piecewise linear functions, which will result in a mixed integer linear program (MILP).

For transparency of the linearization process, we reformulate the VI-constraint (6) as

$$
\sum_{a \in \mathcal{A}}\left(\alpha t_{a}\left(v_{a}\right)+\tau_{a}\right)\left(v_{a}-\hat{v}_{a}^{s}\right) \leq 0, s \in 1 \ldots S
$$

in which the travel cost functions have been separated into travel time functions and link tolls, and are specified as a sum over all links. 
Without loss of generality we add the bound $V_{a}$ on the link flow $v_{a}$ for all $a \in \mathcal{A}$, which can be relaxed by setting $V_{a}$ high enough, and introduce the variables $\bar{t}_{a} \geq t_{a}\left(v_{a}\right) v_{a}$ and $r_{a} \geq \tau_{a} v_{a}$. The TDP can then be expressed as

$$
\begin{aligned}
& \text { P-TDP: } \min _{\{\tau, \bar{t}, r, v, y\} \in \Theta} F_{\mathrm{TDP}}(\tau, \bar{t}, r, v, y)=\alpha \sum_{a \in A} \bar{t}_{a}+\sum_{a \in A} g_{a}\left(v_{a}, y_{a}\right) \\
& \text { where } \\
& \Theta= \begin{cases}\sum_{a \in \mathcal{A}}\left(\alpha \bar{t}_{a}+r_{a}-\left(\alpha t_{a}\left(v_{a}\right)+\tau_{a}\right) \hat{v}_{a}^{s}\right) \leq 0, & s \in 1 \ldots S \\
\bar{t}_{a}=t_{a}\left(v_{a}\right) v_{a}, & a \in A \\
r_{a}=\tau_{a} v_{a}, & a \in A \\
v_{a} \leq V_{a}, & a \in A \\
(\tau, y) \in \Phi, & \\
v \in \Omega . & \end{cases}
\end{aligned}
$$

We will now proceed with the MILP approximation of the TDP. The approximation will be performed in such a way that the resulting MILP formulation gives a lower bound estimation of the optimal objective function value of P-TDP. To simplify the presentation, we will assume that $g(v, y)$ is a linear function, but the same framework can be extended to non-linear functions. The travel time, total travel time and link toll revenue functions are separable for each link. For each link and function we need to specify the approximation by adding additional continuous and binary variables, as well as constraints, in order to transform P-TDP into a MILP.

Remark 3.1. Let $\Theta_{\mathrm{TDP}}$ and $\Theta_{\mathrm{MILP}}$ be the feasible region of P-TDP and its MILP approximation respectivly, and $F(x)$ the objective function of P-TDP. If $\Theta_{\mathrm{TDP}} \subseteq \Theta_{\mathrm{MILP}}$, then $\min \left\{F(x): x \in \Theta_{\mathrm{TDP}}\right\} \geq \min \left\{F(x): x \in \Theta_{\mathrm{MILP}}\right\}$.

Considering the feasible region defined in (9), note that if the total travel time and link toll revenues are underestimated and the travel time is overestimated, then $\Theta_{\text {TDP }} \subseteq \Theta_{\text {MILP }}$. Based on Remark 3.1 we can then conclude that the optimal objective function value of the MILP approximation will be a lower bound estimation of the optimal objective function value of P-TDP. We will now proceed to define the under and over estimations used in the MILP approximation.

\subsection{Approximation of travel time function}

The travel time function, $t_{a}\left(v_{a}\right)$, will be approximated by the piecewise linear function

$$
\phi_{a}=\max _{m \in 1 \ldots M} d_{a, m} v_{a}+e_{a, m}
$$

where $M$ is the number of linear segments, and the parameters $d_{a, m}$ and $e_{a, m}$ are chosen to give an overestimation of the travel time. The overestimation ensures 
that the MILP approximation will give a relaxation of the TDP. In Figure 1, an example of a travel time function and its piecewise linear overestimation is illustrated. Each linear segment is specified by the link flow, $K_{a, m-1} \leq$ $v_{a} \leq K_{a, m}$. The linear segments are computed as the linear function between $\left(K_{a, m-1}, t_{a}\left(K_{a, m-1}\right)\right)$ and $\left(K_{a, m}, t_{a}\left(K_{a, m}\right)\right)$, for $m \geq 1 . K_{a, 0}$ correspond to the flow at $v_{a}=0$, i.e. $t_{a}\left(K_{a, 0}\right)$ is the free flow travel time, and we require that $K_{a, M} \geq V_{a}$.

Assuming that, $t_{a}\left(v_{a}\right)$ is convex over the feasible set $\Omega, d_{a, m}$ and $e_{a, m}$ are chosen as

$$
\left\{\begin{array}{l}
d_{a, m}=\frac{t_{a}\left(K_{a, m}\right)-t_{a}\left(K_{a, m-1}\right)}{K_{a, m}-K_{a, m-1}} \\
e_{a, m}=t_{a}\left(K_{a, m-1}\right)-K_{a, m-1} \frac{t_{a}\left(K_{a, m}\right)-t_{a}\left(K_{a, m-1}\right)}{K_{a, m}-K_{a, m-1}}
\end{array}\right.
$$

Any choice of $d_{a, m}$ and $e_{a, m}$ which ensure that $\phi_{a} \geq t_{a}\left(v_{a}\right)$ for any feasible link flow, relaxes the assumption of $t_{a}\left(v_{a}\right)$ being convex. If the travel time function is piecewise linear in its original form it can be used with the formulation presented here. In that case it will, however, not be an approximation but an exact formulation of the travel time function. Note that the total travel time and link toll revenue still need to be approximated.

Proposition 3.1. For $v_{a}=v_{a}^{*}$, the piecewise linear function (10) with parameters given by (11) yields an overestimation of $t_{a}\left(v_{a}^{*}\right)$.

Proof. Note that $d_{a, m^{*}} v_{a}-e_{a, m^{*}}$ is a line passing through $\left(K_{a, m^{*}-1}, t_{a}\left(K_{a, m^{*}-1}\right)\right)$ and $\left(K_{a, m^{*}}, t_{a}\left(K_{a, m^{*}}\right)\right)$, with $K_{a, m^{*}-1} \leq v_{a}^{*} \leq K_{a, m^{*}}$. Since $t_{a}\left(v_{a}\right)$ is convex, $d_{a, m^{*}} v_{a}^{*}-e_{a, m^{*}} \geq t_{a}\left(v_{a}^{*}\right)$. Any other line $d_{a, m} v_{a}-e_{a, m}$ passing through $\left(K_{a, m-1}, t_{a}\left(K_{a, m-1}\right)\right)$ and $\left(K_{a, m}, t_{a}\left(K_{a, m}\right)\right), m \neq m^{*}$ must therefore satisfy $d_{a, m} v_{a}^{*}-e_{a, m} \leq t_{a}\left(v_{a}^{*}\right)$. By the definition (10) of the piecewise linear function, $\phi_{a}$ must give an overestimation of $t_{a}\left(v_{a}^{*}\right)$.

Even though the travel time function is convex, we actually have a minus in front of the travel time in the VI-constraints in (9), and thus $-t_{a}\left(v_{a}\right)$ is concave. This means that standard approaches for modeling convex piecewise linear functions cannot be used for $t_{a}\left(v_{a}\right)$. We will instead adopt a common formulation of non-convex piecewise linear functions (Dantzig, 1963) and the properties of this formulation are further discussed by Padberg (2000). Consider the set 


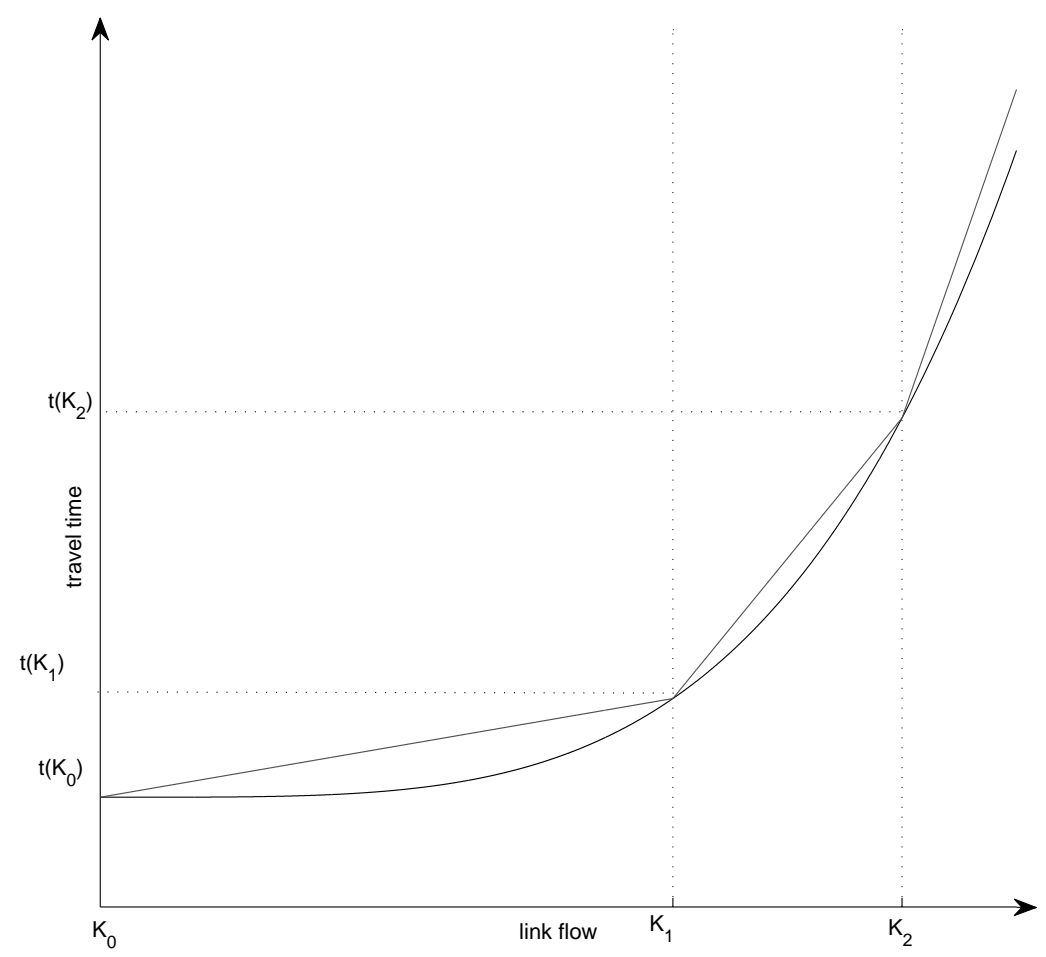

Figure 1: Example of a travel time function and its piecewise linear overestimation

of constraints

$$
\begin{cases}v_{a}=\sum_{m=1}^{M} \rho_{a, m} & \\ \phi_{a}=t_{a}(0)+\sum_{m=1}^{M} d_{a, m} \rho_{a, m} & \\ \rho_{1} \leq K_{a, 1} & \\ \rho_{a, m} \geq\left(K_{a, m}-K_{a, m-1}\right) \omega_{a, m}, & m \in 1 \ldots M-1 \\ \rho_{a, m+1} \leq\left(K_{a, m+1}-K_{a, m}\right) \omega_{a, m}, & m \in 1 \ldots M-1 \\ \rho_{a, m} \geq 0, & m \in 1 \ldots M \\ \omega_{a, m} \in\{0,1\}, & m \in 1 \ldots M-1 .\end{cases}
$$

Proposition 3.2. The set of constraints and variables (12) is a model of the piecewise linear function (10) with parameters given by (11). 
Proof. For convenience the index $a$ is dropped. First we note that the inequalities in (12) ensure that $\omega_{m+1} \leq \omega_{m}$, for all $m \in 1 \ldots . M-1$. From (12) we also have that $\omega_{m}=0 \Leftrightarrow \rho_{m+1}=0$, and that $\omega_{m}=1 \Leftrightarrow \rho_{m}=K_{m}$.

We will now proceed to prove that the case when $\omega_{m^{*}}=1$ and $\omega_{m^{*}+1}=0$ correspond to having a flow $v$ in the interval $K_{m^{*}} \leq v \leq K_{m^{*}+1}$ and $\phi=$ $d_{m^{*}+1} v+e_{m^{*}+1}$. If $\omega_{m^{*}+1}=0$ then $\omega_{k}=0$ for $k \in[m+2, M-1]$ and thus $\rho_{k}=0$, and if $\omega_{m}^{*}=1$ then $w_{l}=1$ for $l \in\left[1, m^{*}\right]$ with $\rho_{l}=K_{l}-K_{l-1}$. If we insert $\omega_{m^{*}}=1$ and $\omega_{m^{*}+1}=0$ in (12) it becomes clear that $0 \leq \rho_{m^{*}+1} \leq$ $K_{m^{*}+1}-K_{m^{*}}$, and that $\rho_{l}=K_{l}-K_{l-1}$ for every $l \in\left[1, m^{*}\right] . v=\sum_{m=1}^{M} \rho_{m}$ implies that $K_{m^{*}} \leq v \leq K_{m^{*}+1}$ and the link travel time can then be expressed as $\phi=t(0)+\sum_{m=1}^{m^{*}} d_{m}\left(K_{m}-K_{m-1}\right)+d_{m^{*}+1} \rho_{m^{*}+1}$. Since $d_{m}$ is the slope of the $m^{\text {th }}$ linear segment we have $t(0)+\sum_{m=1}^{m^{*}} d_{m}\left(K_{m}-K_{m-1}\right)=t\left(K_{m^{*}}\right)$, and we also have $\rho_{m^{*}+1}=v-K_{m^{*}}$. The link travel time can then be expressed as $\phi=t\left(K_{m^{*}}\right)-K_{m^{*}} d_{m^{*}+1}+d_{m^{*}+1} v_{=} d_{m^{*}+1} v+e_{m^{*}+1}$. Following the same argumentation it is easy to see that for the case when $\omega_{1}=0$ we must have $0 \leq v \leq K_{1}$ with $\phi=d_{1} v+e_{1}$, and if $\omega_{M-1}=1$ then $K_{M-1} \leq v \leq K_{M}$ with $\phi=d_{M} v+e_{M}$.

\subsection{Approximation of total travel time function}

The total link travel time function, $t_{a}\left(v_{a}\right) v_{a}$ is assumed to be convex over the feasible set $\Omega$. Note that by applying the same approach for the approximation of the total travel time function as for the travel time function, the assumption of convexity can be relaxed. The assumption of convexity is, however, not restrictive for commonly used travel time functions, e.g. the BPR-function (Bureau of Public Roads, 1964).

The total link travel time function is approximated by the piecewise linear function

$$
\bar{t}_{a}=\max _{n \in 1 \ldots N} \bar{d}_{a, n} v_{a}+\bar{e}_{n, a},
$$

where $N$ is the number of linear segments, and the parameters $\bar{d}_{a, n}$ and $\bar{e}_{a, n}$ are chosen to give an underestimation of the total travel time and thus guarantee a relaxation of P-TDP. Each segment will be a first-order linear approximation of $t_{a}\left(v_{a}\right) v_{a}$ in the point $v_{a}=L_{a, n}$, and $\bar{d}_{a, n}$ and $\bar{e}_{a, n}$ can be expressed as

$$
\left\{\begin{array}{l}
\bar{d}_{a, n}=\left.\frac{\partial t_{a}\left(v_{a}\right) v_{a}}{\partial v_{a}}\right|_{L_{a, n}} \\
\bar{e}_{a, n}=t_{a}\left(L_{a, n}\right) L_{a, n}-\left.L_{a, n} \frac{\partial t_{a}\left(v_{a}\right) v_{a}}{\partial v_{a}}\right|_{L_{a, n}}
\end{array}\right.
$$

Since the total travel time function is convex the piecewise linear function can be transformed into the set of linear inequalities

$$
\bar{t}_{a} \geq \bar{d}_{a, n} v_{a}+\bar{e}_{n, a}, \quad n \in 1 \ldots N .
$$




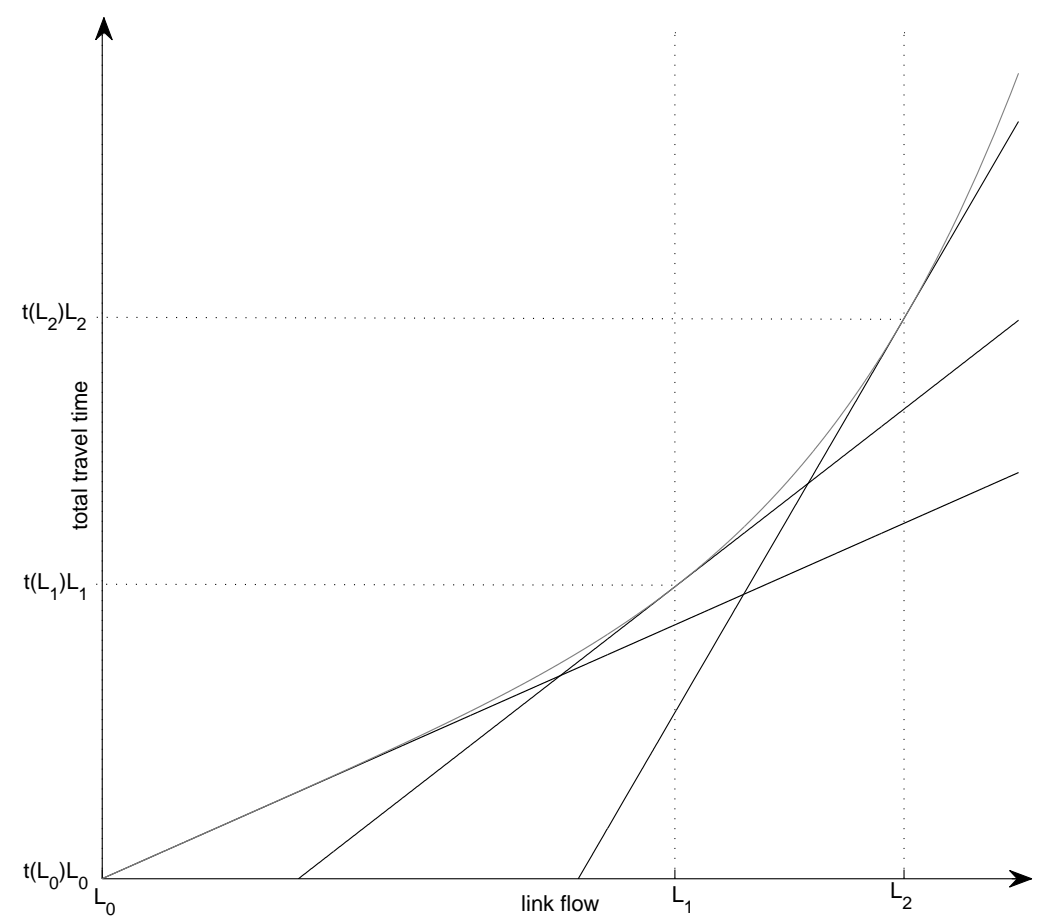

Figure 2: Example of a total travel time function and a set of linear inequalities which give an underestimation of this function

For an example of the total travel time function and the set of linear inequalities which underestimate it, see Figure 2.

Proposition 3.3. Replacing constraints $\bar{t}_{a}=t_{a}\left(v_{a}\right) v_{a}$ in (9) with (13) gives a relaxation of $\Theta$.

Proof. $t_{a}\left(v_{a}\right) v_{a}$ is convex and the linear inequalities in (13) are first-order approximations of $t_{a}\left(v_{a}\right) v_{a}$ for different values on $v_{a}$. Thus (13) must give a relaxation of $\Theta$ since $t_{a}\left(v_{a}\right) v_{a} \geq \bar{d}_{a, n} v_{a}+\bar{e}_{n, a}$ for any $n \in 1 \ldots N$.

\subsection{Approximating the link toll revenue function}

The link toll revenue function for link $a$ is $\tau_{a} v_{a}$, which is a bilinear term. The bilinear term can be underestimated by its convex envelope (McCormick, 1976; Al-Khayyal and Falk, 1983). An underestimation of the link toll revenue function will ensure that that the feasible region of P-TDP is not reduced. The convex envelope for a bilinear term is specified by the lower and upper bounds 
of the link flows and toll levels. Karuppiah and Grossmann (2006) and Meyer and Floudas (2006) further improve the approximation of bilinear terms by applying different convex envelopes for different regions of the feasible area. This approximation, which takes the form of a MILP, is further generalized and discussed by Wicaksono and Karimi (2008).

We will divide the link flow space into $P$ segments, defined by the link flow break points $J_{a, p}, p \in 1 \ldots P$. The convex envelope for a link $a$, with flow $J_{a, p-1} \leq v_{a} \leq$ $J_{a, p}$ is specified as

$$
\left\{\begin{array}{l}
r_{a} \geq v_{a} \tau^{U}+\tau_{a} J_{a, p}-J_{a, p} \tau_{a}^{U} \\
r_{a} \geq v_{a} \tau^{L}+\tau_{a} J_{a, p-1}-J_{a, p-1} \tau_{a}^{L},
\end{array}\right.
$$

where $\tau_{a}^{L}$ and $\tau_{a}^{U}$ are the lower and upper bounds of the toll level on link $a$ respectively. Setting $\tau_{a}^{L}=0$ for every link $a$ gives

$$
\left\{\begin{array}{l}
r_{a} \geq v_{a} \tau^{U}+\tau_{a} J_{a, p}-J_{a, p} \tau_{a}^{U} \\
r_{a} \geq \tau_{a} J_{a, p-1} .
\end{array}\right.
$$

Note that when the link flow on link $a$ is equal to any of the link flow break points $J_{a, p}$, or the toll levels are equal to 0 or $\tau_{a}^{U}$, the approximation given by the convex envelope is equal to the bilinear term. Assume that for a specific $v_{a}$ and $\tau_{a}, r_{a}=v_{a} \tau^{U}+\tau_{a} J_{a, p}-J_{a, p} \tau_{a}^{U}$. Then we can express any link flow as $v_{a}=J_{a, p}-\epsilon_{v}$ and any toll level as $\tau_{a}=\tau_{a}^{U}-\epsilon_{\tau}$. The error, i.e. $\tau_{a} v_{a}-r_{a}$ will then be $\epsilon_{v} \epsilon_{\tau}$. Thus, whenever either $\epsilon_{v}$ or $\epsilon_{\tau}$ tends to zero, the error will also tend to zero.

The underestimation of the link toll revenue applied in this paper is based on the convex hull formulation in Wicaksono and Karimi (2008). We will however use the same approach for determining the active segment at the current link flow as when approximating the travel time functions. This gives the possibility to reduce the number of binary variables and constraints if the link flow segments for approximating the travel time and link toll revenue functions are chosen to be equal, i.e. $M=P$, and for every link $a, J_{a, p}=K_{a, m}$.

For $p \in 1 \ldots P$ we have $J_{a, p-1} \leq v_{a} \leq J_{a, p}$, with $J_{a, 0}=0$ and $J_{a, P} \geq V_{a}$. For each link $a$ we introduced the partial link flow variables $\psi_{a, p}, p \in 1 \ldots P$, and the binary variables $\kappa_{a, p}, p \in 1 \ldots P-1$, to determine which segment is active. Note that $\psi$ and $\kappa$ are equivalent to $\rho$ and $\omega$ in (12). For each segment we will also add an additional toll variable $\gamma_{a, p}, p \in 1 \ldots P$. Let $p^{*}$ denote the active segment, then $\gamma_{a, p}=\tau_{a}$ if $p=p^{*}$, and $\gamma_{a, p}=0$ if $p \neq p^{*}$.

The approximation of the link toll revenue function is for each $a \in \mathcal{A}$ given by 


$$
\begin{cases}v_{a}=\sum_{p=1}^{P} \psi_{a, p} & \\ \psi_{a, 1} \leq J_{a, 1} & \\ \psi_{a, p} \geq\left(J_{a, p}-J_{a, p-1}\right) \kappa_{a, p}, & p \in 1 \ldots P-1 \\ \psi_{a, p+1} \leq\left(J_{a, p+1}-J_{a, p}\right) \kappa_{a, p}, & p \in 1 \ldots P-1 \\ \tau_{a}=\sum_{p=1}^{P} \gamma_{a, p} & \\ \gamma_{a, 1} \leq \tau_{a}^{U}\left(1-\kappa_{a, 1}\right) & \\ \gamma_{a, P} \leq \tau_{a}^{U} \kappa_{a, P-1} & \\ \gamma_{a, p} \leq \tau_{a}^{U}\left(\kappa_{a, p-1}-\kappa_{a, p}\right) & \\ r_{a} \geq v_{a} \tau_{a}^{U}+\sum_{p=1}^{P} J_{a, p} \gamma_{a, p} & \\ \quad-\tau_{a}^{U}\left(J_{a, 1}\left(1-\kappa_{a, 1}\right)+\sum_{p=2}^{P-1} J_{a, p}\left(\kappa_{a, p-1}-\kappa_{a, p}\right)+J_{a, P} \kappa_{a, P-1}\right) \\ r_{a} \geq \sum_{p=1}^{P} J_{a, p-1} \gamma_{a, p} & \\ \psi_{a, p}, \gamma_{a, p} \geq 0, & p \in 1 \ldots P \\ \kappa_{a, p} \in\{0,1\}, & p \in 1 \ldots P-1 .\end{cases}
$$

Proposition 3.4. For any link flow $J_{a, p^{*}-1} \leq v_{a} \leq J_{a, p^{*}}$, and toll level $\tau_{a}<$ $\tau_{a}^{U}$, (15) will give the convex envelope (14).

Proof. For convenience the index $a$ is dropped. Note that any vector of $\kappa$ satisfying (15) must satisfy $\kappa_{p+1} \leq \kappa_{p}$ for all $p \in 1 \ldots . P-1$. Following the proof of Proposition 3.2 it can easily be shown that $\kappa_{p^{*}}=1$ and $\kappa_{p^{*}+1}=0$ correspond to having a flow $v$ in the interval $J_{p^{*}-1} \leq v \leq J_{p^{*}}$.

Consider the three different cases, i) $\kappa_{p}=0$ for all $p$, ii) $\kappa_{p}=1$ for all $p$, and iii) $\kappa_{p}=1$ for all $p<p^{*}$ and $\kappa_{p}=0$ for all $p \geq p^{*}$. Any feasible choice of values for $\kappa$ must fall into exactly one of these three cases.

i) If $\kappa_{p^{*}}=0$ for $p^{*}=1$, then $\kappa_{p}=0$ for any $p$, and from (15) it follows that $\gamma_{p^{*}} \leq \tau^{U}$ and $\gamma_{p}=0$ for any $p>1$.

ii) If $\kappa_{p^{*}}=1$ for $p^{*}=P-1$, then $\kappa_{p}=1$ for any $p$, and from (15) it follows that $\gamma_{p^{*}} \leq \tau^{U}$ and $\gamma_{p}=0$ for any $p<P-1$.

iii) If $\kappa_{p^{*}-1}=1$ and $\kappa_{p^{*}}=0$, then $\kappa_{p}=1$ for all $p<p^{*}$, and $\kappa_{p}=0$ for all $p \geq p^{*}$. Thus $\gamma_{p^{*}} \leq \tau^{U}$, and $\gamma_{p}=0$ for any $p<p^{*}$ and $p>p^{*}$.

We have now shown that for $p^{*}$ we will have $\gamma_{p^{*}}>0$ and $\gamma_{p}=0$ for $p \neq p^{*}$. Thus we must have $\tau=\sum_{p=1}^{P} \gamma_{p}=\gamma_{p^{*}}$, and from (15) we get the convex envelope $r \geq v \tau^{U}+J_{p^{*}} \tau-\tau^{U} J_{p^{*}}, r \geq J_{p^{*}-1} \tau$. 
Proposition 3.5. Replacing constraint $r_{a}=\tau_{a} v_{a}$ in (9) with (15) gives a relaxation of $\Theta$.

Proof. The proof follows directly from Proposition 3.4 and Theorem 2 in AlKhayyal and Falk (1983). For $J_{a, p-1} \leq v_{a} \leq J_{a, p}$ the convex envelope is given by (15). Theorem 2 in Al-Khayyal and Falk (1983) proves that the convex envelope (14) gives an underestimation of $v_{a} \tau_{a}$ for $v_{a} \in\left[J_{a, p-1}, J_{a, p}\right]$ and $\tau \in\left[0, \tau_{a}^{U}\right]$. Thus (15) gives a relaxation of $r_{a}=\tau_{a} v_{a}$ in $\Theta$.

\subsection{A combined approximation of travel time and link toll revenue}

If the same segmentation of the link flow space is applied for both the approximation of the travel time and the link toll revenue functions, the total number of binary variables and constraints can be reduced. Assume that $M=P$ and $K_{a, m}=J_{a, m}$ for every link $a$, then (12) and (15) would give $\rho_{a, m}=\psi_{a, m}$, and $\omega_{a, m}=\kappa_{a, m}$. Replacing $\psi$ and $\kappa$ with $\rho$ and $\omega$ in (15) let us reduce (12) and (15) to

$$
\left\{\begin{array}{l}
v_{a}=\sum_{m=1}^{M} \rho_{a, m} \\
\phi_{a}=t_{a}(0)+\sum_{m=1}^{M} d_{a, m} \rho_{a, m} \\
\tau_{a}=\sum_{m=1}^{M} \gamma_{a, m} \\
\rho_{a, 1} \leq K_{a, 1} \\
\rho_{a, m} \geq\left(K_{a, m}-K_{a, m-1}\right) \omega_{a, m}, \quad m \in 1 \ldots M-1 \\
\rho_{a, m+1} \leq\left(K_{a, m+1}-K_{a, m}\right) \omega_{a, m}, \quad m \in 1 \ldots M-1 \\
\gamma_{a, 1} \leq \tau_{a}^{U}\left(1-\omega_{a, 1}\right) \\
\gamma_{a, M} \leq \tau_{a}^{U} \omega_{a, M-1} \\
\gamma_{a, M} \leq \tau_{a}^{U}\left(\omega_{a, m-1}-\omega_{a, m}\right) \\
r_{a} \geq v_{a} \tau_{a}^{U}+\sum_{p=1}^{P} K_{a, p} \gamma_{a, p} \\
\quad-\tau_{a}^{U}\left(K_{a, 1}\left(1-\omega_{a, 1}\right)+\sum_{m=2}^{M-1} K_{a, m}\left(\omega_{a, m-1}-\omega_{a, m}\right)+K_{a, M} \omega_{a, M-1}\right) \\
r_{a} \geq \sum_{m=1}^{M} K_{a, m-1} \gamma_{a, m} \\
\gamma_{a, m}, \rho_{a, m} \geq 0, \\
\omega_{a, m} \in\{0,1\},
\end{array}\right.
$$




\subsection{The MILP formulation}

If the operator cost function is convex, it can be approximated in a similar way as the total travel time, and if it contains non-convex parts it can be approximated in a similar way as the travel time function. One special case appears when $g_{a}\left(v_{a}, y_{a}\right)=C_{a} v_{a} y_{a}$, where $C_{a}$ is a cost associated with each passing vehicle (transaction cost). Then $v_{a} y_{a}$ can be reformulated by its convex envelope. Note that since $y_{a}$ either takes on the value of 0 or 1 , the convex envelope will give an exact reformulation, and not an approximation. By introducing additional binary variables it is also possible to have an operator cost function which is not link-additive, e.g. for every $n$ located tolls there is a fixed cost. For the remaining part of this paper we will, however, assume that the operator cost function is given by the affine function $g_{a}\left(v_{a}, y_{a}\right)$.

Based on the piecewise linear approximations of the non-linear functions in the TDP, its MILP approximation is formulated as

$$
\text { P-MILP: } \min _{\gamma, \kappa, \xi, \rho, \tau, \phi, \psi, \omega, r, \bar{t}, v, y} F_{\mathrm{MILP}}(\bar{t}, v, y)=\sum_{a \in \mathcal{A}}\left(\alpha \bar{t}_{a}+g_{a}\left(v_{a}, y_{a}\right)\right)
$$

subject to

1) the VI-constraints:

$$
\sum_{a \in \mathcal{A}}\left(\alpha \bar{t}_{a}+r_{a}\right)-\sum_{a \in \mathcal{A}}\left(\alpha \phi_{a}+\tau_{a}\right) \hat{v}_{a}^{s} \leq 0, s \in 1 \ldots S
$$

2) the feasible link flows and toll levels:

$$
\left\{\begin{array}{l}
(\tau, y) \in \Phi \\
v_{a} \leq V_{a}, \quad a \in \mathcal{A} \\
v \in \Omega
\end{array}\right.
$$

For all $a \in \mathcal{A}$ :

3) the approximation of travel time given by (12)

4) the approximation of total travel time given by (13)

5) the approximation of link toll revenue given by (15)

Note that alternatively (12) in 3) and (15) in 5), can be replaced by (16).

Lemma 3.1. P-MILP is a relaxation of P-TDP.

Proof. The relaxation of $\Theta$, from the introduction of the piecewise linearization of the total travel time function and link toll revenue functions, follows from Proposition 3.3 and Proposition 3.5. Furthermore, Proposition 3.1 together with 
3.2 states that (12) gives an overestimation $\phi_{a}$ of $t_{a}\left(v_{a}\right)$. Note that the sign is negative in front of $t_{a}\left(v_{a}\right)$ in the VI-constraints of the TDP, and thus

$$
\sum_{a \in \mathcal{A}}\left(\alpha \bar{t}_{a}+r_{a}-\left(\alpha \phi_{a}+\tau_{a}\right) \hat{v}_{a}^{s}\right) \leq \sum_{a \in \mathcal{A}}\left(\alpha \bar{t}_{a}+r_{a}-\left(\alpha t_{a}\left(v_{a}\right)+\tau_{a}\right) \hat{v}_{a}^{s}\right)
$$

for any $(\tau, \phi, \bar{t}, v$,$) which satisfies (12),(13),(15),(17)$ and (18). Thus we have concluded that the feasible region of P-TDP is relaxed, and since the objective function in P-TDP is unchanged, P-MILP is a relaxation of P-TDP.

Theorem 3.1. An optimal solution to P-MILP gives a lower bound estimation of the optimal solution to P-TDP.

Proof. Let $x$ and $x^{\prime}$ be the optimal solution to P-TDP and P-MILP respectively. Then $F_{\mathrm{MILP}}\left(x^{\prime}\right) \leq F_{\mathrm{MILP}}(x) \leq F_{\mathrm{TDP}}(x)$. The first inequality holds because $x$ is a feasible solution to P-MILP, and the second inequality holds because of Lemma 3.1 .

\subsection{Extending the MILP formulation}

While the MILP approximation is presented for a fixed demand, single user class network, and with additive tolls, the formulation can be extended to elastic demand, multiclass networks and non-additive tolls. Dafermos (1980a) present the VI formulation of the more general elastic demand, multiclass traffic equilibrium problem, which can be used to formulate a MILP approximation with elastic demand and/or multiclass networks. For more details on these formulations we refer to Sheffi (1984) or Patriksson (1994).

Elastic demand will require piecewise linearization of functions related to travel demand. There will be three functions related to travel demand, which are all related to the inverse travel demand function in each OD pair; the inverse travel demand function, the negative of the integral of the inverse travel demand function and the negative of the inverse travel demand function times the demand. If these functions are convex, then the piecewise linear approximation can be done without adding additional binary variables, in the same way as the total travel time functions are approximated (see Section 3.2). For non-convex functions the piecewise linear approximation require introduction of additional binary variables (see Section 3.1).

If the difference between user classes are limited to a different value of time for each class, the main difference in the MILP approximation is that not only the total link flow space needs to be divided into different segments, but also the link flow space for each user class. Thus, the number of binary variables will increase. For multiple user classes together with elastic demand, the number of functions related to the demand which need to be approximated, will grow proportional to the number of user classes. 
The possibility to use non-additive tolls, i.e. non-linear pricing schemes (Maruyama and Sumalee, 2007; Lawphongpanich and Yin, 2012), is an interesting extension to the TDP formulated in this paper, since most congestion pricing schemes implemented in practice include some component of non-linear pricing. One special case of non-linear pricing is an area pricing scheme. In such a scheme the road users are charged, for instance once a day, and are then allowed to drive freely for the rest of the day within the charged area. For the case of area pricing, the optimization problem is somewhat different compared to the one presented in this paper, since what links to toll and their corresponding toll levels are no longer the decision variables, but what links should belong to the charged area, and what level to charge the road users driving within the area. We will not go into the details of the formulation of the UE problem for this case, and the interested reader is referred to Maruyama and Sumalee (2007). Extending the MILP approximation presented in this paper to cases of non-linear pricing is straight forward as long as the corresponding UE problem is formulated based on link flows (e.g. area pricing). For the case of area pricing, the toll revenues will be approximated for each area instead of for each link, the approximation of the travel time and total travel time functions will, however, remain the same.

\section{Solution algorithm}

\subsection{A cutting constraint algorithm}

Any solver capable of solving mixed integer linear programs can be used to solve P-MILP. The VI-constraints, however, are specified for each extreme point of $\Omega$, and the number of extreme points can be expected to be large. To generate the complete set of VI-constraints is not only computationally burdensome but also unnecessary, since we do not expect all the VI-constraints to be binding at optimum. In the solution algorithm, we therefore solve a relaxed version of P-MILP, with only a subset of the VI-constraints included, and generate the VI-constraints iteratively.

The relaxed MILP, with a reduced number, $R$, of extreme points is

$$
\begin{gathered}
\text { P-MILP-R: } \min _{\gamma, \kappa, \xi, \rho, \tau, \phi, \psi, \omega, r, \bar{t}, v, y} F_{\mathrm{MILP}}(\bar{t}, v, y)=\sum_{a \in \mathcal{A}}\left(\alpha \bar{t}_{a}+g_{a}\left(v_{a}, y_{a}\right)\right) \\
\text { subject to } \sum_{a \in \mathcal{A}}\left(\alpha \bar{t}_{a}+r_{a}\right)-\sum_{a \in \mathcal{A}}\left(\alpha \phi_{a}+\tau_{a}\right) \hat{v}_{a}^{s} \leq 0 \quad s \in 1 \ldots R \\
\quad \text { and constraints (12), (13), (15) and (18). }
\end{gathered}
$$

Let $\phi^{*}, \tau_{a}^{*}, r_{a}^{*}$ and $\bar{t}_{a}^{*}$ be the optimal solution to P-MILP-R with $R \leq S$. If there exists a solution $\tilde{v} \in \Omega$ which yields $\sum_{a \in \mathcal{A}}\left(\alpha \bar{t}_{a}+r_{a}\right)-\sum_{a \in \mathcal{A}}\left(\alpha \phi_{a}+\tau_{a}\right) \hat{v}_{a}^{s}>0$ then $\tilde{v}$ is an extreme point of $\Omega$ which if added to the set of VI-constraints would 
make the current solution $\mathbf{z}$ infeasible. Searching for such an extreme point is equivalent to solving the linear program:

$$
\text { P-LP: } \max _{\bar{v} \in \Omega} F_{\mathrm{LP}}(\tilde{v})=\sum_{a \in \mathcal{A}}\left(\alpha \bar{t}_{a}^{*}+r_{a}^{*}\right)-\sum_{a \in \mathcal{A}}\left(\alpha \phi_{a}^{*}+\tau_{a}^{*}\right) \tilde{v}_{a} .
$$

If $\sum_{a \in \mathcal{A}}\left(\alpha \bar{t}_{a}^{*}+r_{a}^{*}\right)-\sum_{a \in \mathcal{A}}\left(\alpha \phi_{a}^{*}+\tau_{a}^{*}\right) \tilde{v}_{a} \leq 0$ then there exists no additional $\tilde{v} \in \Omega$ which would make the current solution to P-MILP-R infeasible, and the current solution is thus the optimal solution to P-MILP. On the other hand, if the optimal solution to P-LP, $\tilde{v}$, gives $\sum_{a \in \mathcal{A}}\left(\alpha \bar{t}_{a}^{*}+r_{a}^{*}\right)-\sum_{a \in \mathcal{A}}\left(\alpha \phi_{a}^{*}+\tau_{a}^{*}\right) \tilde{v}_{a}>$ 0 , then $\tilde{v}$ is a new extreme point that needs to be added to P-MILP-R. The new extreme point will generate a new VI-constraint for which the current solution to P-MILP-R is not feasible.

Note that if we remove the constant part from P-LP we have

$$
\min _{\bar{v} \in \Omega} F_{\mathrm{LP}}(\tilde{v})=\sum_{a \in \mathcal{A}}\left(\alpha \phi_{a}+\tau_{a}\right) \tilde{v}_{a},
$$

which is an uncapacitated network flow problem, and can be solved by finding the shortest path in every OD pair.

The MILP cutting constraint algorithm (CCA) can be outlined as

Step 0. Initialization Set the iteration counter $k:=1$, and find an initial set of extreme points, denoted $\Gamma^{k}$.

Step 1. $P$-MILP-R Solve P-MILP-R with the set of extreme points $\Gamma^{k}$, which gives link travel times $\phi^{k}$, link total travel time $\bar{t}^{k}$, link flows $v^{k}$, link toll levels $\tau^{k}$, and link toll revenues $r^{k}$.

Step 2. P-LP Solve P-LP, with the solution from P-MILP-R as input. The optimal solution to P-LP is $\tilde{v}^{k}$.

Step 3. Convergence check If $\sum_{a \in \mathcal{A}}\left(\alpha \bar{t}_{a}^{k}+r_{a}^{k}\right)-\sum_{a \in \mathcal{A}}\left(\alpha \phi_{a}^{k}+\tau_{a}^{k}\right) \tilde{v}_{a} \leq 0$ then terminate the algorithm.

Step 4. Extreme point set update Let the solution to P-LP, $\tilde{v}^{k}$ be the new extreme point, i.e. set $\Gamma^{k+1}:=\Gamma^{k} \cup\left\{\tilde{v}^{k}\right\}$, and set $k:=k+1$. Go to Step 1.

If a good set of initial extreme points are used in Step 0, the number of times which P-MILP-R needs to be solved can be reduced. This is further discussed in the numerical results.

The following proposition is trivial and only stated to complete the development of the algorithm.

Proposition 4.1. Let $z^{*}$ denote the final global optimal solution found by the MILP cutting constraint algorithm, then $z^{*}$ also solves P-MILP. 
Proof. In Step 1, P-MILP-R is a mixed integer linear program which can be solved to its global optimum, with optimal solution $z_{k}^{*}$. Thus at each iteration, $k, z_{k}$ must be the global optimal solution to P-MILP-R. In Step 3, the algorithm will only terminate if $\sum_{a \in \mathcal{A}}\left(\alpha \bar{t}_{a}+r_{a}\right)-\sum_{a \in \mathcal{A}}\left(\alpha \phi_{a}+\tau_{a}\right) \tilde{v}_{a} \leq 0$, where $\tilde{v}$ is the solution to P-LP, and thus $z^{*}$ must also be the global optimal solution to P-MILP.

Proposition 4.2. The MILP CCA converges in a finite number of iteration.

Proof. The algorithm will terminate if either (i) no other extreme point can be found to make the current solution infeasible, or (ii) all extreme points are generated and included in the problem. For (i), this implies that the algorithm will converge in a finite number of iterations. For (ii), since the number of extreme points of $\Omega$ is finite, the algorithm will also converge in a finite number of iterations in this situation.

Proposition 4.3. The optimal objective function of $P-M I L P-R$ will not decrease with each iteration of the MILP CCA, i.e. $F_{\mathrm{P}-\mathrm{MILP}-\mathrm{R}}\left(z_{k}^{*}\right) \leq F_{\mathrm{P}-\mathrm{MILP}-\mathrm{R}}\left(z_{k+1}^{*}\right)$, with $z_{k}^{*}$ being the optimal solution to P-MILP-R in iteration $k$.

Proof. In each iteration of the MILP CCA a new VI-constraint is added, making the current global optimal solution infeasible. Thus $F_{\mathrm{P}-\mathrm{MILP}-\mathrm{R}}\left(z_{k}^{*}\right)>F_{\mathrm{P} \text {-MILP-R }}\left(z_{k+1}^{*}\right)$, cannot hold since the solution to P-MILP-R in iteration $k+1$ is also feasible in iteration $k$.

\subsection{Requirements on the solution accuracy of P-MILP-R}

MILP-P is merely an approximation, which has the property of global optimality, of P-TDP. The computational effort for solving P-MILP partly depends on the accuracy of the approximated travel time, total travel time and link toll revenue functions. A tradeoff between solving P-MILP to its optimality and to approximate $\mathrm{P}-\mathrm{TDP}$ with high accuracy is therefore needed.

When solving a MILP, the concept of branch and bound, first introduced by Land and Doig (1960), is usually adopted. In theory a branch and bound approach solves any MILP, in the worst case by exhaustively searching of all possible integer solutions. In most industrial solvers (e.g. CPLEX, Gurobi), the approach of branch and bound is used together with heuristic algorithms for finding good feasible solutions. The main deficiency with this approach is that even if the optimal solution is quickly obtained, it may be computational burdensome and sometimes not possible to verify that this solution is optimal. It is therefore of interest to discuss the case when the solution to P-MILP or rather P-MILP-R is not guaranteed to be the optimal one.

First we will consider the case when P-MILP-R is not solved to optimality in the first $n$ iterations of the MILP CCA. Proposition 4.2 will still hold, since in each iteration a new extreme point is still generated, and the algorithm 
converges in a finite number of iterations. Proposition 4.3 will, on the other hand, not hold for the first $n$ iterations, i.e. the optimal objective function value of P-MILP-R may not increase in each iteration. Since P-MILP-R is solved to optimality after $n$ iterations, Proposition 4.3 will hold for all iterations for which $k>n$. This result can be practically used by first applying the MILP CCA with heuristic solutions to P-MILP-R, and then applying the MILP CCA, given the previously generated VI-constraints, with optimal solutions to P-MILP-R. If the final heuristic solution to P-MILP-R is the optimal one, only one additional iteration with the MILP CCA is required to prove optimality. If not, further iterations may be necessary.

The second case is when P-MILP-R is not solved to its optimality, in the intermediate and final iterations of the MILP CCA . Then Proposition 4.1 will not hold and there is no guarantee that the solution found is the optimal solution to P-MILP. It may not even be an underestimation of the optimal objective function value to P-TDP. Still, the solution satisfies the VI-constraints and can therefore be considered to give an approximation of the user equilibrium link flows and travel times, given the toll level solution from P-MILP. If the heuristic used for solving P-MILP-R and the accuracy of the approximated piecewise linear functions are good, the solution obtained from P-MILP may be useful in practice even though the optimality of the solution cannot be verified.

\subsection{Iteratively updating the linearization scheme}

To reduce the error, which is introduced by the approximation of the total travel time, travel time, and link toll revenue functions, the link flow solution from MILP CCA can be used to improve the approximation. The updated approximation will be calculated for the link flow $v_{a}^{\mathrm{MILP}}$ on a link $a$, which is optimal in P-MILP. If the flow $v_{a}^{\mathrm{MILP}}$ do not correspond to one of the break points (or points of linearization for the case of total travel time) in the piecewise linearization scheme it can be added as a new break point (or point of linearization). The updated approximation will then give $\bar{t}_{a}=t_{a}\left(v_{a}^{\mathrm{MILP}}\right) v_{a}^{\mathrm{MILP}}, \phi_{a}=t_{a}\left(v_{a}^{\mathrm{MILP}}\right)$ and $r_{a}=\tau_{a} v_{a}^{\mathrm{MILP}}$, which clearly will reduce the feasible area of P-MILP. If we denote the initial approximation scheme as $l_{1}$ and the updated one as $l_{2}$, and denote their corresponding optimal solutions to P-MILP as $z_{l_{1}}^{*}$ and $z_{l_{2}}^{*}$ respectively, then $F_{\mathrm{MILP}}\left(z_{l_{1}}^{*}\right) \leq F_{\mathrm{MILP}}\left(z_{l_{2}}^{*}\right)$ must hold.

Given a new approximation scheme, the MILP CCA is run once again and a new solution is obtained. In our numerical experiments this strategy has shown to give good approximations, but will on the other hand increase the computational time. A tradeoff between the number of improved approximation schemes and the computational time is inevitable. Note that the VI-constraints from the previous MILP CCA iterations should be used as initial VI-constraints when restarting the algorithm with an updated approximation scheme. 


\section{Numerical results}

For the numerical results the nine node network from Hearn and Ramana (1998), with 18 links and four OD pairs, and the Sioux Falls network from Yildirim (2001), with 87 links and 30 OD pairs, are used. For both networks the travel time functions follow the BPR-form:

$$
t_{a}\left(v_{a}\right)=t_{a}^{0}\left(1+0.15\left(\frac{v_{a}}{\mu_{a}}\right)^{4}\right),
$$

where $t_{a}^{0}$ is the free flow travel time, and $\mu_{a}$ the link capacity for link $a$. The value of time is assumed to be equal to one, i.e. $\alpha=1$. For the numerical results presented here, without loss of generality, the toll-point cost for link $a, g_{a}$, is assumed to be flow independent, and is given by

$$
g_{a}\left(y_{a}\right)=C y_{a} .
$$

For the approximation schemes used in these numerical results, the same points in link flow space are used to specify the MILP approximations of both the travel time, total travel time, and link toll revenue functions, which reduce (12) and (15) to (16). The approximation scheme will be iteratively updated, with the initial scheme denoted as $l_{1}$ with link flows $v\left(l_{1}\right)$. Adding the link flow solution $v\left(l_{n}\right)$ to the current approximation scheme give us $l_{n+1}$, and by rerunning the MILP CCA with the updated approximation scheme a new solution can be obtained.

The MILP formulation will be used to compute system optimal (SO) (with all links tollable) and UE solutions (no links tollable). These solutions are denoted MILP-SO and MILP-UE respectively. The MILP-SO link flow solution and objective function value will only depend on the approximation of the total travel time functions, even though the approximation of the travel time and link toll revenue functions will influence the toll levels. The MILP-UE link flow solution and objective function value will depend on both the approximation of the total travel time and travel time functions, but not on the approximation of the link toll revenue functions. The total link flow error, $e$, is computed as

$$
e=\sum_{a \in \mathcal{A}}\left|v_{a}^{\mathrm{MLP}}-v_{a}^{*}\right|,
$$

where $v^{*}$ is the real $\mathrm{SO}$ or $\mathrm{UE}$ link flows.

When possible, the toll solution from P-MILP will be compared with the known optimal solution to P-TDP, but this is usually only possible if the $y$-variables are fixed and the number of tolled links are small. Any known feasible solution to P-TDP can however be used as an upper bound (UBD) of the objective function value. The relative gap between objective function value of P-MILP, $F_{\text {MILP }}$, and an UBD is measured as

$$
G A P=\frac{U B D-F_{\mathrm{MILP}}}{U B D} .
$$


P-MILP-R and P-LP are solved by Gurobi version 3.0, running on an AMD Opteron(tm) 285 Quad core processor. Gurobi supports multiprocessing, but all computational times are given in CPU-seconds. To reduce the computational time, a set of initial VI-constraints are generated by first solving the MILP-SO problem followed by the MILP-UE problem.

The Gurobi solver incorporates both a heuristic solver for finding good feasible solutions and a branch and bound algorithm for proving optimality. For larger networks, the computational burden required to prove optimality can be substantial, or it might not even be possible to prove the optimality of the solution. Thus, by setting Gurobi to focus on finding good feasible solutions (with the heuristic solver) rather than trying to prove optimality, the computational time can be significantly reduced. Solutions whose optimality is not proven will be considered as heuristic. If the heuristic solution in fact is the optimal one, only one additional iteration with the MILP CCA is required to prove optimality. This strategy is used for the Sioux Falls network. The practical problem then becomes how to set the termination criteria in Gurobi. For the results presented here a fixed time limit is used to terminate the Gurobi solver, which unfortunately has to be given in clock time and not in CPU-seconds. Since a quad core processor is used for most of the computations, the time limit, in CPU-seconds, is approximately four times the clock time. The time limit is a rough termination criterion, and for many cases the optimal solution will be found much faster. Finally, when proving optimality, the branch and bound process will terminate when the relative gap (denoted as mipgap in Gurobi) between the lower bound in the branch and bound process and the best found integer solution is small enough. For the experiments presented in this paper the mipgap parameter is set to $1 \mathrm{e}-6$.

\subsection{The nine node network}

The network layout of the nine node network is presented in Figure 3, with the network data in Table 1. An initial approximation scheme is specified by 14 link flow points for each link, given as fractions of the link capacities in an interval of 0.2 , starting at 0.0 , up to 2.2 , and in an interval of 0.3 , between 2.2 and 2.8. The upper bound on the link flows and toll levels are set to $V_{a}=\min \left\{60,2.8 * \mu_{a}\right\}$ and $\tau_{a}^{U}=20$ respectively. For each scenario the MILP CCA is repeated ten times, each time with an improved approximation scheme.

In Table 1 and Table 2, the SO/MILP-SO and UE/MILP-UE link flows are compared respectively, and in Table 3, the total link flow errors $e$ and the objective function value, $F$, are given for $l_{1}$ to $l_{10}$. In general, a toll level solution which support the SO flow is not unique and in Table 1 one particular SO toll level solution is presented, namely the minimum toll booth (MTB) solution from Hearn and Ramana (1998), which is the solution with the minimum number of tolled links (see the MTB column in Table 1). When the approximation is improved, it can be seen in Table 3 that the P-MILP objective function value 


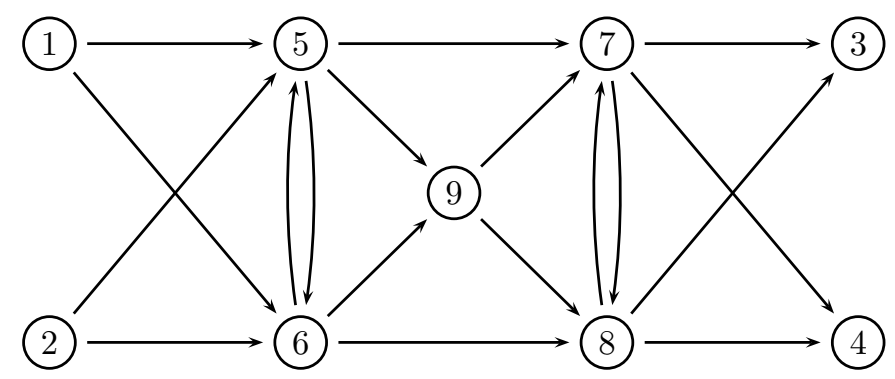

Figure 3: The nine node network

is increased and tends towards that of P-TDP. The link flow errors, however, is not necessarily reduced each time a new link flow solution is added to the approximation scheme (e.g. $l_{2}$ and $l_{3}$ for the UE case). Considering the errors for the ten consecutive approximation schemes, the error still seems to tend towards zero, in this example. For $l_{10}$ there is almost a perfect match between the objective function values of P-TDP and P-MILP. The MILP-SO and MILPUE solution gives a total of 36 initial VI-constraints.

We will now proceed and present results for the toll design problem. The MTB solution of five tolled links, presented in Table 1, gives us an upper bound on the number of tolled links. Five different toll-point costs of $0.1,5,10,50$ and 100 have been used and results are presented for each one. The optimal objective function values from P-MILP, $F_{\text {MILP }}$, is given in Table 4 , and applying the link toll solution to P-TDP gives $F_{\text {TDP }}$, which is presented in Table 5 . For each approximation scheme the relative gap between $F_{\text {MILP }}$ and the best found feasible solution is given in Table 6 , and the link toll solution for the best found solution is given in Table 7 .

For all toll-point costs it is obvious that $F_{\text {MILP }}$ is increased whenever the approximation scheme is improved. For $C=0.1$ and $C=5$ the best solutions (with almost zero relative gap) correspond to the MTB toll locations, and for $C=0.1$ the relative gap is below $1 \%$ for all approximation schemes. With $C=5$ the relative gap is above $1 \%$ for $l_{1}$, and less than $1 \%$ for the following approximation schemes. Comparing the $C=0.1$ and $C=5$ columns in Table 7 with the MTB solution in Table 1, there is only a small difference between the toll level solutions. We note that the relative gap is smaller for low toll-point costs, and when the toll point cost is increased, the relative gap is increased. The computational times in Table 8 are increased when moving from $C=0.1$ to $C=5$ and $C=10$, and then reduced for $C=50$ and $C=100$. It is clear that the computational time is affected by the toll-point cost, and there are two reasons for this result. When the toll-point cost is increased, there will be fewer toll locations to consider in practice, which will improve the performance of the branch and bound procedure and thus reduce the computational time. When the toll-point cost is close to zero on the other hand, the optimal objective func- 
Table 1: Network data, SO link flows, and MTB toll levels for the nine node network

\begin{tabular}{|c|c|c|c|c|c|c|}
\hline Link & $t_{a}^{0}$ & $k_{a}$ & $v^{*}$ & $v\left(l_{1}\right)$ & $v\left(l_{10}\right)$ & $M T B$ \\
\hline $1-5$ & 5 & 12 & 9.41 & 10.04 & 9.41 & 0 \\
\hline $1-6$ & 6 & 18 & 20.59 & 19.96 & 20.59 & 0 \\
\hline $2-5$ & 3 & 35 & 38.33 & 38.11 & 38.33 & 4.00 \\
\hline $2-6$ & 9 & 35 & 31.67 & 31.89 & 31.67 & 0 \\
\hline $5-6$ & 9 & 20 & 0.00 & 0.00 & 0.00 & 0 \\
\hline $5-7$ & 2 & 11 & 21.30 & 20.96 & 21.30 & 11.20 \\
\hline $5-9$ & 8 & 26 & 26.44 & 27.19 & 26.44 & 0 \\
\hline $6-5$ & 4 & 11 & 0.00 & 0.00 & 0.00 & 0 \\
\hline $6-8$ & 6 & 33 & 39.47 & 36.60 & 39.47 & 7.20 \\
\hline $6-9$ & 7 & 32 & 12.78 & 15.25 & 12.78 & 0 \\
\hline $7-3$ & 3 & 25 & 29.61 & 28.97 & 29.61 & 4.00 \\
\hline $7-4$ & 6 & 24 & 20.76 & 20.83 & 20.76 & 0 \\
\hline $7-8$ & 2 & 19 & 0.00 & 0.00 & 0.00 & 0 \\
\hline $8-3$ & 8 & 39 & 10.39 & 11.03 & 10.39 & 0 \\
\hline $8-4$ & 6 & 43 & 39.24 & 39.17 & 39.24 & 0 \\
\hline $8-7$ & 4 & 36 & 0.00 & 0.00 & 0.00 & 0 \\
\hline $9-7$ & 4 & 26 & 29.06 & 28.83 & 29.07 & 3.20 \\
\hline $9-8$ & 8 & 30 & 10.16 & 13.61 & 10.15 & 0 \\
\hline$F$ & - & - & 2253.92 & 2239.11 & 2253.92 & - \\
\hline$e$ & - & - & 0.00 & 13.24 & 0.044 & - \\
\hline
\end{tabular}

tion value is close to the SO solution. The LP-relaxation of P-MILP, which is solved in the initial branch and bound node, will always give the SO objective function value, and if the toll-point cost is low, fewer iteration with the branch and bound process are needed to prove the optimality.

The computational times, for solving P-MILP for each approximation scheme, are given in Table 8. When the approximation scheme is improved, the problem size, in terms of binary variables and constraints, is increased. Improving the approximation scheme far from the current solution will have no effect on the solution, and only a small effect on the computational time. Adding "good" points of approximation, e.g. adding the current link flow solution to the specification of the approximation scheme, will have a large impact on the solution, but will also increase the computational time. Comparing the computational times for $l_{8}$ and $l_{9}$, for $C=10$, the computational time is actually decreased when the approximation is improved. For $l_{8}$ the heuristic solver finds the optimal solution fast, which reduces the number of nodes in the branch and bound process, while for $l_{9}$ the heuristic solver is unable to find the optimal solution, which is obtained instead from one of the last evaluated branch and bound nodes.

Even though the computational times are high for some of the improved approximation schemes, good solutions are already found with the first few ap- 
Table 2: UE solutions for the nine node network

\begin{tabular}{|c|c|c|c|}
\hline Link & $v^{*}$ & $v\left(l_{1}\right)$ & $v\left(l_{10}\right)$ \\
\hline $1-5$ & 8.16 & 9.80 & 8.16 \\
\hline $1-6$ & 21.84 & 20.20 & 21.84 \\
\hline $2-5$ & 47.37 & 43.49 & 47.37 \\
\hline $2-6$ & 22.63 & 26.51 & 22.63 \\
\hline $5-6$ & 0.00 & 0.00 & 0.00 \\
\hline $5-7$ & 27.84 & 27.68 & 27.84 \\
\hline $5-9$ & 27.69 & 25.61 & 27.69 \\
\hline $6-5$ & 0.00 & 0.00 & 0.00 \\
\hline $6-8$ & 44.47 & 43.15 & 44.47 \\
\hline $6-9$ & 0.00 & 3.56 & 0.00 \\
\hline $7-3$ & 38.16 & 35.05 & 38.16 \\
\hline $7-4$ & 17.37 & 21.47 & 17.38 \\
\hline $7-8$ & 0.00 & 0.00 & 0.00 \\
\hline $8-3$ & 1.84 & 4.95 & 1.85 \\
\hline $8-4$ & 42.63 & 38.53 & 42.62 \\
\hline $8-7$ & 0.00 & 0.00 & 0.00 \\
\hline $9-7$ & 27.69 & 28.83 & 27.69 \\
\hline $9-8$ & 0.00 & 0.33 & 0.00 \\
\hline$F$ & 2455.87 & 2379.59 & 2455.79 \\
\hline$e$ & 0.00 & 34.04 & 0.030 \\
\hline
\end{tabular}

proximation schemes. For example, for $C=0.1$ the optimal toll location (but not the toll level solution) is already obtained with $l_{5}$, and for $C=5$ with $l_{3}$. For the toll-point costs equal to 50 and 100, the optimal solutions are known, which are to toll link 5-7 with the level of 8.0 and to toll no links, respectively. The best toll location solutions to P-MILP, for both a toll-point cost of 50 and 100, correspond to the known optimal toll locations. For $C=50$ the toll level solution from P-MILP for link 5-7 is 7.83, which is close to the known global optimal solution to P-TDP.

It is also important to point out that while $F_{\text {MILP }}$ increases when the approximation is improved, the quality of the toll solution, i.e. $F_{\mathrm{TDP}}$, is not necessarily improved. This effect is related to how the approximation scheme is updated. It is the current optimal link flow solution which is introduced as a new break point in the approximation scheme. Thus, the objective function value around the current solution is increased (due to the improved approximation of the non-linear functions). Then there is the possibility that a solution, far away from the optimal one, at which the approximation gives a large underestimation of the objective function of P-TDP, becomes the minimum of P-MILP. An example of this effect can be seen if we study the case when $C=100$. For $l_{2}$ the solution to P-MILP is to toll no links, with the objective function value 2391.88. Forcing $y_{6}$ to be positive (which will be the optimal toll location with 
Table 3: Total link flow error, $e$, and objective function value, $F$, for the MILP-SO and MILP-UE solutions

\begin{tabular}{|c|c|c|c|c|}
\hline & \multicolumn{2}{|c|}{$M I L P-S O$} & \multicolumn{2}{c|}{$M I L P-U E$} \\
Approximation scheme & $e$ & $F$ & $e$ & $F$ \\
\hline$l_{1}$ & 13.23 & 2239.11 & 34.04 & 2379.59 \\
\hline$l_{2}$ & 8.22 & 2252.23 & 17.56 & 2413.85 \\
\hline$l_{3}$ & 3.36 & 2253.54 & 19.07 & 2431.29 \\
\hline$l_{4}$ & 1.19 & 2253.74 & 7.17 & 2437.64 \\
\hline$l_{5}$ & 1.07 & 2253.87 & 4.93 & 2448.05 \\
\hline$l_{6}$ & 0.53 & 2253.91 & 1.51 & 2452.02 \\
\hline$l_{7}$ & 0.15 & 2253.91 & 3.18 & 2454.25 \\
\hline$l_{8}$ & 0.15 & 2253.92 & 0.20 & 2455.35 \\
\hline$l_{9}$ & 0.11 & 2253.92 & 0.05 & 2455.74 \\
\hline$l_{10}$ & 0.04 & 2253.92 & 0.03 & 2455.79 \\
\hline
\end{tabular}

Table 4: Optimal objective function value to P-MILP, $F_{\mathrm{MILP}}$

\begin{tabular}{|c|c|c|c|c|c|}
\hline & \multicolumn{5}{|c|}{ Toll-point cost } \\
Approximation scheme & 0.1 & 5 & 10 & 50 & 100 \\
\hline$l_{1}$ & 2239.51 & 2254.43 & 2269.43 & 2326.47 & 2376.47 \\
\hline$l_{2}$ & 2252.79 & 2272.07 & 2287.07 & 2346.20 & 2391.88 \\
\hline$l_{3}$ & 2254.04 & 2274.25 & 2290.10 & 2364.94 & 2405.18 \\
\hline$l_{4}$ & 2254.24 & 2275.38 & 2294.15 & 2376.44 & 2421.54 \\
\hline$l_{5}$ & 2254.37 & 2277.80 & 2295.85 & 2378.09 & 2429.07 \\
\hline$l_{6}$ & 2254.41 & 2278.31 & 2297.44 & 2385.78 & 2430.60 \\
\hline$l_{7}$ & 2254.41 & 2278.83 & 2297.60 & 2388.14 & 2433.43 \\
\hline$l_{8}$ & 2254.42 & 2278.9 & 2300.86 & 2392.56 & 2435.82 \\
\hline$l_{9}$ & 2254.42 & 2278.91 & 2301.46 & 2394.32 & 2438.17 \\
\hline$l_{10}$ & 2254.42 & 2278.92 & 2303.44 & 2394.44 & 2439.43 \\
\hline
\end{tabular}

$l_{3}$ ) and solving P-MILP to find the optimal value of $\tau_{6}$ for $l_{2}$ gives the objective function value 2396.2. Now the optimal link flow solution from $l_{2}$ is introduced as break points in the approximation scheme, and the new optimal solution is to toll link $6\left(y_{6}=1\right)$ with a toll level of 6.89 and an optimal objective function value of 2405.18. Computing objective function value of P-MILP for the zero toll level solution from $l_{2}$, but with the $l_{3}$ approximation scheme gives an objective function value of 2423.16 . When changing from $l_{2}$ to $l_{3}$, the objective function value for the no toll solution has increased from 2391.88 to 2423.16, while the objective function value when tolling link 6 has changed from 2396.2 to 2405.18 and become the optimal solution. 
Table 5: $F_{\mathrm{TDP}}$, given the optimal toll level solution from P-MILP

\begin{tabular}{|c|c|c|c|c|c|}
\hline & \multicolumn{5}{|c|}{ Toll-point cost } \\
Approximation scheme & 0.1 & 5 & 10 & 50 & 100 \\
\hline$l_{1}$ & 2301.63 & 2298.54 & 2313.54 & 2415.71 & 2465.71 \\
\hline$l_{2}$ & 2320.29 & 2345.73 & 2360.73 & 2414.49 & 2455.87 \\
\hline$l_{3}$ & 2268.58 & 2303.53 & 2312.67 & 2411.22 & 2463.66 \\
\hline$l_{4}$ & 2258.39 & 2303.86 & 2317.62 & 2413.65 & 2463.55 \\
\hline$l_{5}$ & 2254.43 & 2345.43 & 2324.29 & 2447.47 & 2461.37 \\
\hline$l_{6}$ & 2256.66 & 2306.06 & 2314.34 & 2415.22 & 2455.87 \\
\hline$l_{7}$ & 2254.62 & 2284.26 & 2365.39 & 2422.83 & 2504.68 \\
\hline$l_{8}$ & 2254.45 & 2278.93 & 2328.13 & 2411.25 & 2472.81 \\
\hline$l_{9}$ & 2254.42 & 2278.92 & 2314.10 & 2442.88 & 2465.14 \\
\hline$l_{10}$ & 2254.42 & 2278.95 & 2311.97 & 2411.54 & 2461.17 \\
\hline
\end{tabular}

Table 6: The relative gap (in \%) between $F_{\mathrm{MILP}}$ and the best found solution for each toll point cost in Table 5

\begin{tabular}{|c|c|c|c|c|c|}
\hline & \multicolumn{5}{|c|}{ Toll-point cost } \\
Approximation scheme & 0.1 & 5 & 10 & 50 & 100 \\
\hline$l_{1}$ & 0.66 & 1.07 & 1.84 & 3.52 & 3.23 \\
\hline$l_{2}$ & 0.07 & 0.30 & 1.08 & 2.70 & 2.61 \\
\hline$l_{3}$ & 0.02 & 0.21 & 0.95 & 1.92 & 2.06 \\
\hline$l_{4}$ & $<0.01$ & 0.16 & 0.77 & 1.44 & 1.40 \\
\hline$l_{5}$ & $<0.01$ & 0.049 & 0.70 & 1.37 & 1.09 \\
\hline$l_{6}$ & $<0.001$ & 0.027 & 0.63 & 1.06 & 1.03 \\
\hline$l_{7}$ & $<0.001$ & $<0.01$ & 0.62 & 0.96 & 0.91 \\
\hline$l_{8}$ & $<0.0001$ & $<0.001$ & 0.48 & 0.77 & 0.82 \\
\hline$l_{9}$ & $<0.0001$ & $<0.001$ & 0.45 & 0.70 & 0.72 \\
\hline$l_{10}$ & $<0.0001$ & $<0.0001$ & 0.37 & 0.70 & 0.67 \\
\hline
\end{tabular}

\subsection{The Sioux Falls network}

The version of the Sioux Falls network used in this paper was first introduced in Yildirim (2001), and the network layout is presented in Figure 4. The network has 87 links, 30 nodes and 30 OD-pairs, of which 11 links are connectors to the origin and destination nodes, and will not be considered as tollable. In its original form, the network from Yildirim (2001) has elastic demand elastic. For the numerical results presented in this paper the demand will, however, be considered as fixed, and set to be the demand level of the system optimal solution reported in Yildirim (2001). The minimum number of tolls needed to reach the SO link flow solution has been computed by the method presented in Hearn and Ramana (1998) (denoted as MTB solution), which result in tolls on 13 links (Table 13). Note that the MTB solution is not guaranteed to be unique, and there can exist other solution with the same objective function values but 
Table 7: The link toll solution with lowest $F_{\text {TDP }}$

\begin{tabular}{|c|c|c|c|c|c|}
\hline & \multicolumn{5}{|c|}{ Toll-point cost (approximation scheme) } \\
Link & $0.1\left(l_{9}\right)$ & $5\left(l_{9}\right)$ & $10\left(l_{10}\right)$ & $50\left(l_{3}\right)$ & $100\left(l_{2}, l_{6}\right)$ \\
\hline $1-5$ & 0 & 0 & 0 & 0 & 0 \\
\hline $1-6$ & 0 & 0 & 0 & 0 & 0 \\
\hline $2-5$ & 4.01 & 4.01 & 3.99 & 0 & 0 \\
\hline $2-6$ & 0 & 0 & 0 & 0 & 0 \\
\hline $5-6$ & 0 & 0 & 0 & 0 & 0 \\
\hline $5-7$ & 11.20 & 11.20 & 8.34 & 7.83 & 0 \\
\hline $5-9$ & 0 & 0 & 0 & 0 & 0 \\
\hline $6-5$ & 0 & 0 & 0 & 0 & 0 \\
\hline $6-8$ & 7.20 & 7.20 & 0 & 0 & 0 \\
\hline $6-9$ & 0 & 0 & 0 & 0 & 0 \\
\hline $7-3$ & 4.00 & 4.00 & 0 & 0 & 0 \\
\hline $7-4$ & 0 & 0 & 0 & 0 & 0 \\
\hline $7-8$ & 0 & 0 & 0 & 0 & 0 \\
\hline $8-3$ & 0 & 0 & 0 & 0 & 0 \\
\hline $8-4$ & 0 & 0 & 4.01 & 0 & 0 \\
\hline $8-7$ & 0 & 0 & 0 & 0 & 0 \\
\hline $9-7$ & 3.18 & 3.18 & 0 & 0 & 0 \\
\hline $9-8$ & 0 & 0 & 0 & 0 & 0 \\
\hline
\end{tabular}

with tolls located on other links.

Similar to the nine node network, the parameters for the initial approximation scheme is given as a fraction of link capacities, in intervals of 0.3 between 0 and 3.6. The upper bound of the link flows and toll levels are set to $V_{a}=$ $\min \left\{80,3.6 \mu_{a}\right\}$ and $\tau_{a}^{U}=20$ respectively. To illustrate the effect of the number of tollable links on the relative gap and computational times, we will present results, with toll point cost $C=5$, for the case when only the five most congested links are tollable, and when all links are tollable (except for the connectors). For the case when all links are tollable results are provided for the toll point costs $0.5,5,20$ and 100. The computational time will limit the number of improved approximation schemes, and results will be presented for $l_{1}$ to $l_{4}$.

To solve P-MILP more efficiently, the MILP CCA is first applied with a time limit criterion in Gurobi to get a heuristic solution, without actually proving optimality. Secondly the MILP CCA will be repeated, initialized with the heuristic solution and corresponding VI-constraints, with the purpose to prove optimality of the heuristic solution. Increasing the problem size, either by increasing the number of tollable links or by improving the approximation scheme, increases the difficulty to solve P-MILP, and the time limit therefore increases in these cases. The clock time limit criterion is initially set to 150 seconds when limiting the number of tollable links to the five most congested, and to 300 seconds when 


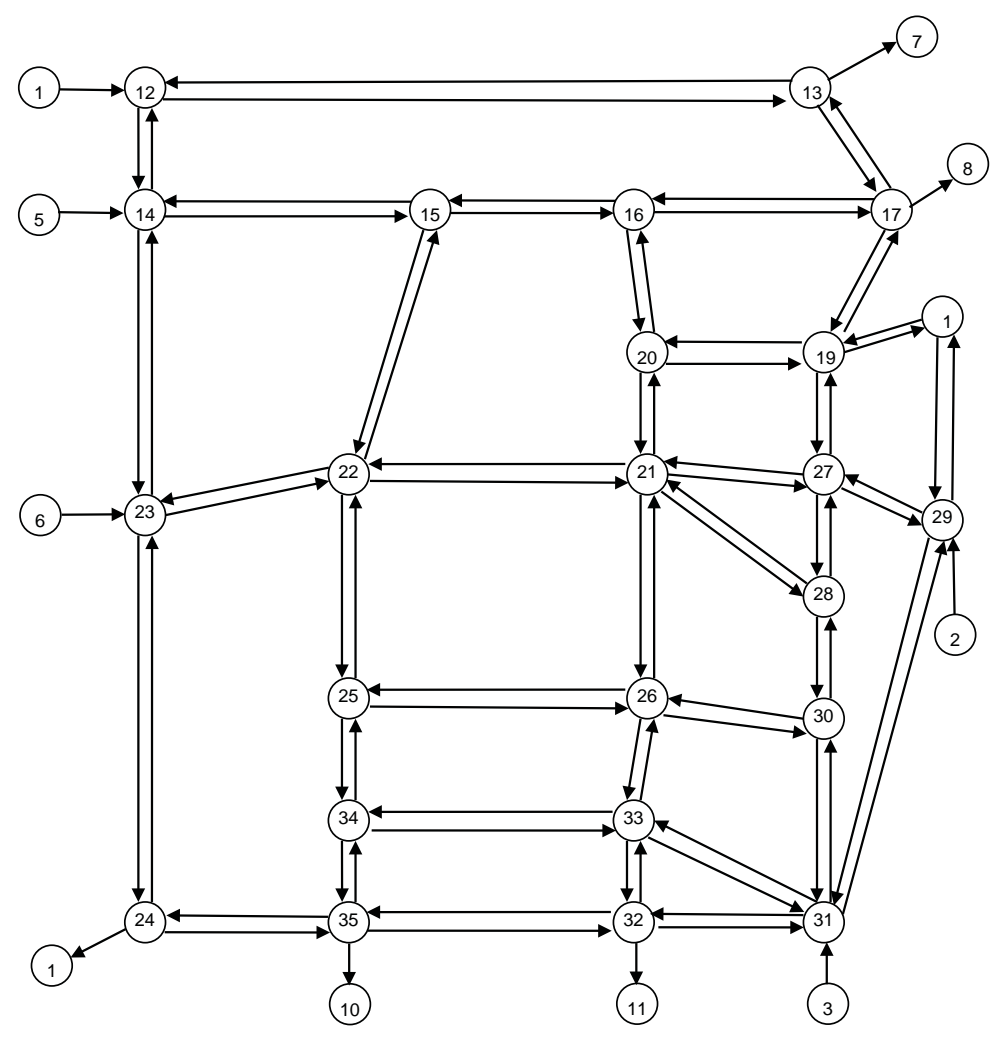

Figure 4: The Sioux Falls network 
Table 8: Computational time in CPU-seconds, with the number of generated VI-constrints in parenthesis

\begin{tabular}{|c|c|c|c|c|c|}
\hline $\begin{array}{c}\text { Approximation } \\
\text { scheme }\end{array}$ & 0.1 & 5 & 10 & 50 & 100 \\
\hline$l_{1}$ & $58(5)$ & $22(0)$ & $57(1)$ & $123(2)$ & $119(4)$ \\
\hline$l_{2}$ & $30(1)$ & $47(0)$ & $64(0)$ & $55(0)$ & $73(0)$ \\
\hline$l_{3}$ & $25(0)$ & $99(0)$ & $132(0)$ & $249(1)$ & $87(0)$ \\
\hline$l_{4}$ & $60(1)$ & $264(0)$ & $398(0)$ & $608(1)$ & $600(3)$ \\
\hline$l_{5}$ & $31(0)$ & $680(0)$ & $422(0)$ & $721(1)$ & $247(0)$ \\
\hline$l_{6}$ & $94(2)$ & $1204(0)$ & $1179(0)$ & $362(0)$ & $366(0)$ \\
\hline$l_{7}$ & $57(0)$ & $3369(1)$ & $2602(0)$ & $370(0)$ & $1102(1)$ \\
\hline$l_{8}$ & $103(0)$ & $5518(1)$ & $6570(0)$ & $681(0)$ & $552(0)$ \\
\hline$l_{9}$ & $154(0)$ & $6440(1)$ & $6099(0)$ & $1273(0)$ & $1197(0)$ \\
\hline$l_{10}$ & $161(0)$ & $3615(0)$ & $33034(0)$ & $1525(0)$ & $420(0)$ \\
\hline
\end{tabular}

all links are tollable. To improve the computational performance, the solution feasibility is preserved within the MILP CCA by resolving P-MILP-R with the new VI-constraint as the objective to minimize, before adding it to the set of VI-constraints. When the tollable links are restricted to the five most congested, $l_{1}$ to $l_{4}$ can be solved to optimality. When all links are tollable $l_{1}$ and $l_{2}$ are solved to optimality, but for $l_{3}$ and $l_{4}$ only heuristic solutions are provided.

The non-linear cutting constraint based algorithm from Lawphongpanich and Hearn (2004) is used to compute local optimal toll levels, given the fixed set of tollable links from the optimal solution to P-MILP. The objective function value corresponding to this solution is denoted $F_{\mathrm{TDP}}^{*}$ with relative gap $G A P^{*}$. Finally, for comparison, the heuristic method based on the smoothening technique in Ekström et al. (2009) is applied to the Sioux Falls network, with corresponding objective function value $F_{\mathrm{S}}$, and the MTB solution is applied with objective function value $F_{\text {мтв }}$.

The MILP-SO and MILP-UE solutions are used to generate 249 initial VIconstraints, and in Table 9 the relative gap between the MILP-SO/UE, and their respective non-linear counterparts are presented, together with the total link flow error $e$. Similar to the result from the nine node network, both the relative gap and the total link flow error is significantly larger for the UE link flow solution, in which both the approximation of travel time and total travel time will affect the link flows, compared with the SO link flow solution which is only affected by the approximation of the total travel time functions.

The cases with the five tollable links, and all tollable links, are presented in Table 10 and Table 11, respectively. Note that the time spent when solving with a time limit depends on the number of VI-constraints which are generated, i.e. the number of iterations with the MILP CCA. If the optimal solution is found within the time limit, verifying optimality with an exact solution will 
only require one iteration with the MILP CCA. All solutions, later proven to be optimal, are found within the time limit. For the case when all links are tollable, the highest obtained lower bound is used for computing the relative gap for those cases when only a heuristic solution is available. For $C=5$, the best solutions obtained by solving P-MILP, have objective functions values at most $1.29 \%$ (with the five most congested links tollable) and $1.21 \%$ (with all links tollable) from the global optimal solution. An important difference between the two scenarios is the number of generated VI-constraints. When the five most congested links are tollable, only four additional VI-constraints need to be generated for $l_{1}$. This can be compared with the case when all links are tollable and 25 VI-constraints are generated. Although the relative gaps are almost similar, the time required to solve P-MILP to optimality is considerable smaller when only five links are tollable. When all links are tollable, optimality can only be proven up to $l_{2}$ but with much higher computational time. For either case it is clear that we need to rely on a heuristic solution to P-MILP-R within the MILP CCA, to be able to solve P-MILP in practice.

An interesting observation is that when comparing the two cases (with the five tollable, and all tollable links), the relative gap is smaller when all links are tollable. This observation is similar to what could be seen in the results for the nine node network in which the relative gap increases when the solution is further away from the SO solution. For the nine node network the solution for a lower toll-point cost was naturally closer to the SO objective compared with a higher toll-point cost. In the Sioux Falls network we expect the objective function value, when all links are tollable, to be equal to or lower than the objective function value when only the five most congested links are tollable. However, one cannot make a general conclusion on this behavior of the MILP approximation based on these two numerical examples.

Comparing $F_{\mathrm{TDP}}, F_{\mathrm{TDP}}^{*}$ and $F_{\mathrm{S}}$, it is clear that the non-linear CCA is capable of improving the solution by optimizing the toll levels, given the toll locations from P-MILP. On the other hand, applying both toll locations and levels from PMILP to P-TDP $\left(F_{\mathrm{TDP}}\right)$ outperform the solution from the smoothening heuristic, and even more so if $F_{\mathrm{TDP}}^{*}$ is considered, for all cases except $C=0.5$. Thus the toll locations from P-MILP appear to be good, while the toll levels can be further improved by a local search method.

In Table 11 the smallest relative gap is presented for each $C$. For the lower bound (LB) the optimal objective function value from P-MILP for $l_{2}$ is used, with the exception of $C=0.5$ for which the $\mathrm{SO}$ objective function value plus 0.5 (since at least one toll needs to be located) is used. For the upper bound (UB) the best found feasible solution is used, which for $C=0.5$ and $C=5$ is the MTB solution, and for $C=20$ and $C=100$ the toll location solution from P-MILP with toll levels given by the non-linear CCA. When $C$ is small enough the MTB solution will be the global optimal solution to P-TDP, and for $C=0.5$ and $C=5$ this solution is the best found one, but with small differences to the other solutions. Continuing to improve the approximation scheme up to 
$l_{8}$ for $C=5$ actually gives $F_{\mathrm{TDP}}^{*}=4330.70$ with 8 located tolls, to compare with $F_{\text {мтв }}=4330.67$ with 13 located tolls.

In Table 13 the MTB solution is compared with solutions to P-MILP with $l_{4}$. For $C=0.5$, seven out of the ten located tolls in the solution to P-MILP are also included in the MTB solution. As the approximation scheme is improved, the number of tolled links are increased, and $F_{\mathrm{TDP}}^{*}$ decreased. For $C=20$ and $C=100$ the combination of toll locations form P-MILP with $l_{4}$, and toll levels from the non-linear CCA, provide the best solution. For all scenarios $F_{\text {MILP }}$ is increased with each improvement of the approximation scheme, $F_{\mathrm{TDP}}$, $F_{\mathrm{TDP}}^{*}$ and $e$ is, however, not necessarily decreased with each improvement of the approximation scheme. Comparing $l_{1}$ with $l_{4}, e$ is clearly decreased for all values on $C$, and the difference between $F_{\mathrm{TDP}}$ and $F_{\mathrm{MILP}}$ as well, suggesting the approximation has been improved.

Table 9: Relative gap and link flow errors for the SO and UE solution

\begin{tabular}{|c|l|c|c|c|c|}
\hline & Approximation scheme & $l_{1}$ & $l_{2}$ & $l_{3}$ & $l_{4}$ \\
\hline \multirow{3}{*}{ SO } & $F_{\text {MILP }}$ & 4238.44 & 4259.80 & 4260.52 & 4262.34 \\
& Gap $(\%)$ & 0.64 & 0.14 & 0.12 & 0.078 \\
& Total link flow error, $e$ & 21.39 & 11.43 & 5.68 & 3.37 \\
\hline \multirow{3}{*}{ UE } & $F_{\text {MILP }}$ & 4405.33 & 4513.46 & 4551.17 & 4568.58 \\
& Gap $(\%)$ & 5.00 & 2.64 & 1.83 & 1.46 \\
& Total link flow error, $e$ & 66.56 & 34.34 & 29.58 & 22.73 \\
\hline
\end{tabular}

Table 10: Results for the Sioux Falls network, only the five most congested links tollable

\begin{tabular}{|l|c|c|c|c|}
\hline Approximation scheme & $l_{1}$ & $l_{2}$ & $l_{3}$ & $l_{4}$ \\
\hline$F_{\mathrm{MILP}}$ & 4262.46 & 4293.06 & 4301.22 & 4306.79 \\
\hline$F_{\mathrm{TDP}}$ & 4372.85 & 4395.01 & 4376.63 & 4375.21 \\
\hline$F_{\mathrm{TDP}}^{*}$ & 4363.27 & 4389.19 & 4368.25 & 4368.25 \\
\hline$F_{\mathrm{S}}$ (from the heuristic algorithm) & \multicolumn{4}{|c|}{4368.25} \\
\hline$G A P(\%)$ & 2.52 & 2.32 & 1.72 & 1.56 \\
\hline$G A P^{*}(\%)$ & 2.31 & 1.61 & 1.42 & 1.29 \\
\hline$e$ & 58.93 & 58.91 & 48.10 & 41.43 \\
\hline CPU-time, time limit & 4076 & 4715 & 8228 & 4719 \\
\hline CPU-time, exact solution & 865 & 2109 & 10254 & 29750 \\
\hline Generated VI constraints & 4 & 3 & 2 & 0 \\
\hline Number of tolled links & 4 & 3 & 5 & 5 \\
\hline
\end{tabular}


Table 11: Results for the Sioux Falls network, all links tollable

\begin{tabular}{|c|c|c|c|c|c|}
\hline$C$ & Approximation scheme & $l_{1}$ & $l_{2}$ & $l_{3}$ & $l_{4}$ \\
\hline \multirow{10}{*}{0.5} & $F_{\mathrm{MILP}}$ & 4240.94 & 4263.64 & 4268.61 & 4270.45 \\
\hline & $F_{\text {TDP }}$ & 4442.15 & 4335.10 & 4300.55 & 4305.78 \\
\hline & $F_{\text {TDP }}^{*}$ & 4377.03 & 4317.92 & 4295.29 & 4291.02 \\
\hline & $F_{\mathrm{S}}\left(F_{\mathrm{MTB}}\right)$ & \multicolumn{4}{|c|}{$4274.67(4272.17)$} \\
\hline & Gap $(\%)$ & 4.53 & 1.65 & 0.83 & 0.93 \\
\hline & $e$ & 88.89 & 61.46 & 36.12 & 41.47 \\
\hline & CPU-time, time limit & 21647 & 2372 & 23625 & 13423 \\
\hline & CPU-time, exact solution & 6102 & 54208 & - & - \\
\hline & Generated VI constraints & 19 & 1 & 6 & 2 \\
\hline & Number of tolled links & 5 & 7 & 9 & 10 \\
\hline \multirow{10}{*}{5} & $F_{\mathrm{MILP}}$ & 4261.34 & 4286.92 & 4292.73 & 4298.90 \\
\hline & $F_{\mathrm{TDP}}$ & 4464.52 & 4353.24 & 4391.25 & 4339.59 \\
\hline & $F_{\mathrm{TDP}}^{*}$ & 4437.78 & 4341.48 & 4369.32 & 4332.69 \\
\hline & $F_{\mathrm{S}}\left(F_{\mathrm{MTB}}\right)$ & \multicolumn{4}{|c|}{$4358.80(4330.67)$} \\
\hline & Gap $(\%)$ & 4.55 & 1.52 & 2.24 & 1.21 \\
\hline & $e$ & 80.83 & 49.65 & 55.69 & 34.15 \\
\hline & CPU-time, time limit & 31684 & 9532 & 3502 & 19102 \\
\hline & CPU-time, exact solution & 8380 & 45239 & - & - \\
\hline & Generated VI constraints & 25 & 3 & 0 & 3 \\
\hline & Number of tolled links & 4 & 5 & 5 & 6 \\
\hline \multirow{10}{*}{20} & $F_{\mathrm{MILP}}$ & 4307.89 & 4343.79 & 4360.65 & 4365.90 \\
\hline & $F_{\mathrm{TDP}}$ & 4552.32 & 4514.25 & 4485.75 & 4425.19 \\
\hline & $F_{\mathrm{TDP}}^{*}$ & 4466.56 & 4469.32 & 4476.60 & 4423.17 \\
\hline & $F_{\mathrm{S}}\left(F_{\mathrm{MTB}}\right)$ & \multicolumn{4}{|c|}{$4447.77(4525.67)$} \\
\hline & $\operatorname{Gap}(\%)$ & 5.37 & 3.78 & 3.16 & 1.84 \\
\hline & $e$ & 88.71 & 53.31 & 64.02 & 40.58 \\
\hline & CPU-time, time limit & 5594 & 6995 & 10640 & 4701 \\
\hline & CPU-time, exact solution & 10771 & 116084 & - & - \\
\hline & Generated VI constraints & 4 & 2 & 2 & 0 \\
\hline & Number of tolled links & 2 & 3 & 3 & 4 \\
\hline \multirow{10}{*}{100} & $F_{\mathrm{MILP}}$ & 4396.68 & 4450.62 & 4455.59 & 4473.25 \\
\hline & $F_{\mathrm{TDP}}$ & 4537.65 & 4636.04 & 4534.12 & 4568.95 \\
\hline & $F_{\mathrm{TDP}}^{*}$ & 4526.56 & 4636.04 & 4526.56 & 4526.56 \\
\hline & $F_{\mathrm{S}}\left(F_{\mathrm{MTB}}\right)$ & \multicolumn{4}{|c|}{$4626.54(5565.67)$} \\
\hline & Gap $(\%)$ & 3.11 & 3.99 & 1.84 & 2.59 \\
\hline & $e$ & 52.37 & 55.12 & 38.02 & 37.57 \\
\hline & CPU-time, time limit & 5488 & $-^{\mathrm{a}}$ & 3504 & 4750 \\
\hline & CPU-time, exact solution & 4402 & 77669 & - & - \\
\hline & Generated VI constraints & 3 & 0 & 0 & 0 \\
\hline & Number of tolled links & 1 & 0 & 1 & 1 \\
\hline
\end{tabular}

a No feasible solution found within the time limit 
Table 12: Best found lower $(L B)$ and upper $(U B)$ bound, and the corresponding relative gap (Gap) (in \%) for each toll point cost

\begin{tabular}{|c|c|c|c|}
\hline$C$ & $L B$ & $U B$ & Gap \\
\hline 0.5 & 4266.17 & 4272.17 & 0.14 \\
\hline 5 & 4286.92 & 4330.67 & 1.01 \\
\hline 20 & 4343.79 & 4423.17 & 1.79 \\
\hline 100 & 4450.62 & 4526.56 & 1.68 \\
\hline
\end{tabular}

Table 13: Comparison between the MTB solution and P-MILP $\left(l_{4}\right)$ solution

\begin{tabular}{|l|c|c|c|c|c|}
\hline Link & $M T B$ & $C=0.5$ & $C=5$ & $C=20$ & $C=100$ \\
\hline $13-17$ & 2.4 & 2.6 & - & - & - \\
\hline $17-13$ & 19.2 & 19.2 & - & - & - \\
\hline $17-16$ & 7.2 & 7.1 & - & - & - \\
\hline $18-19$ & 2.4 & - & - & - & - \\
\hline $18-29$ & - & - & 4.6 & - & - \\
\hline $19-17$ & - & - & 19.5 & 19.4 & 11.9 \\
\hline $21-20$ & 0.8 & - & - & - & - \\
\hline $27-21$ & 5.6 & 4.9 & 4.2 & 4.7 & - \\
\hline $27-28$ & 0.8 & - & - & - & - \\
\hline $28-30$ & - & 3.1 & - & - & - \\
\hline $29-18$ & - & 0.9 & - & - & - \\
\hline $31-32$ & 16.8 & 4.9 & 5.3 & 6.2 & - \\
\hline $31-33$ & 4 & 4.0 & 3.6 & 5.1 & - \\
\hline $33-32$ & 12 & - & - & - & - \\
\hline $33-34$ & 1.6 & - & - & - & - \\
\hline $34-35$ & 1.6 & - & - & - & - \\
\hline $35-32$ & 20 & 8.0 & - & - & - \\
\hline
\end{tabular}




\section{Conclusions and further research}

In this paper we have presented a MILP approximation of the TDP which has the global optimality property. There are two applications of this approximation. One is to find the optimal toll locations and corresponding toll levels to the TDP. The toll level solution from the MILP approximation depends on to the approximation scheme, and will not exactly be equal to the optimal solution to the TDP. In general, by adopting a more refined discretization scheme, it is possible to achieve solutions from the MILP approximation which are very close to the optimal solution to the TDP. Applying the non-linear CCA to solve the TDP with the toll location solution obtained from the MILP approximation, further improve the solution. The other application is to find a good lower bound estimation of the objective function value of the TDP.

For the nine node network it has been shown that as the approximation is improved, solutions which are close to the known optimal solution (when available) will be found. The main question is whether the MILP approximation approach is applicable to large networks. Considering the Sioux Falls network, we are able to find solutions which, when evaluated in the TDP, gives objective function values which are at most $1.79 \%$ from the optimal one. The computational time for proving optimality will, however, grow rapidly as the approximations are improved. To better explore the network structure of the MILP, both for heuristic solutions and within the branch and bound procedure, with the objective to decrease the computational time, and/or to apply more accurate approximation schemes, are interesting directions for future research.

To extend the MILP approximation to the case of elastic demand, multiple user groups and non-additive tolls are natural extensions of the method presented in this paper. Traffic networks with travel time functions which are linear at high flows (e.g. above capacity), can be an interesting application since such travel time functions would reduce the number of binary variables needed for the MILP approximation.

\section{Acknowledgments}

This research has been supported by the Hong Kong Research Grant Council (RGC) through the grant PolyU5261/07E and \#616906. The work behind this paper has been possible thanks to the in-coming attachment program of the Hong Kong Polytechnic University which supported a four month visit to Hong Kong for Joakim Ekström. The presentation of this paper benefited from the comments of three anonymous reviewers.

\section{References}

Al-Khayyal, F. A. and J. E. Falk (1983). Jointly constrained biconvex programming. Mathematics of Operations Research 8(2), 273-286. 
Bureau of Public Roads (1964). Traffic Assignment Manual. Washington D.C., USA: U.S. Dept. of commerce, Urban Planning Division.

Dafermos, S. (1980a). The general multimodal network equilibrium problem with elastic demand. Networks 12(1), 57-72.

Dafermos, S. (1980b). Traffic equilibrium and variation inequalities. Transportation Science 14(1), 42-54.

Dantzig, G. B. (1963). Linear Programming and Extensions. Princeton, New Jersey: Princeton University Press.

Ekström, J., L. Engelson, and C. Rydergren (2009). Heuristic algorithms for a second-best congestion pricing problem. NETNOMICS: Economic Research and Electronic Networking 10(1), 85-102.

Gao, Z., J. Wu, and H. Sun (2005). Solution algorithm for the bi-level discrete network design problem. Transportation Research Part B 39(6), 479 - 495.

Hearn, D. W. and M. V. Ramana (1998). Solving congestion toll pricing models. In P. Marcotte and S. Nguyen (Eds.), Equilibrium and Advances in Transportation Modeling, pp. 109-124. the Netherlands: Kulwer Academic Publishers.

Karuppiah, R. and I. E. Grossmann (2006). Global optimization for the synthesis of integrated water systems in chemical processes. Computers 83 Chemical Engineering 30(4), $650-673$.

Knight, F. (1924). Some fallacies in the interpretation of social cost. Quarterly Journal of Economis 38(4), 582-606.

Land, A. H. and A. G. Doig (1960). An automatic method of solving discrete programming problems. Econometrica 28(3), 497-520.

Lawphongpanich, S. and D. W. Hearn (2004). An MPEC approach to secondbest toll pricing. Mathematical Programming 101(1), 33-55.

Lawphongpanich, S. and Y. Yin (2012). Nonlinear pricing on transportation networks. Transportation Research Part C 20(1), 218-235.

Leblanc, L. J. (1975). Algorithm for the discrete network design problem. Transportation Science 9(3), 183-199.

Liu, L. N. and J. F. McDonald (1999). Economic efficiency of second-best congestion pricing schemes in urban highway systems. Transportation Research Part B 33(3), 157-188.

Marchand, M. (1968). A note on optimal tolls in an imperfect environment. Econometrica 36(3-4), 575-581. 
Marcotte, P. (1983). Network optimization with continuous control parameters. Transportation Science 17(2), 181 - 197.

Maruyama, T. and A. Sumalee (2007). Efficiency and equity comparison of cordon- and area-based road pricing schemes using a trip-chain equilibrium model. Transportation Research Part A 41(7), 655-671.

May, A. D., D. S. Milne, S. P. Shepherd, and A. Sumalee (2002). Specification of optimal cordon pricing locations and charges. Transportation Research Records (1812), 60-68.

McCormick, G. (1976). Computability of global solutions to factorable nonconvex programs: Part I -convex underestimating problems. Mathematical Programming 10(2), 147-175.

Meng, Q. and H. Yang (2002). Benefit distribution and equity in road network design. Transportation Research Part B 36(1), 19 - 35.

Meyer, C. A. and C. A. Floudas (2006). Global optimization of a combinatorially complex generalized pooling problem. AIChE Journal 42(3), 1027-1037.

Padberg, M. (2000). Approximating separable nonlinear functions via mixed zero-one programs. Operations Research Letters 27(1), $1-5$.

Patriksson, M. (1994). The Traffic Assignment Problem: Models and Methods. Utrecht: VSP.

Pigou, A. C. (1920). Welth and Welfare. London: MacMillan.

Sheffi, Y. (1984). Urban Transportation Networks: Equilibrium Analysis with Mathematical Programming Methods. New Jersey: Prentice-Hall.

Shepherd, S. and A. Sumalee (2004). A genetic algorithm based approach to optimal toll level and location problems. Networks and Spatial Economics 4(2), $161-179$.

Sumalee, A. (2004). Optimal road user charging cordon design: A heuristic optimization approach. Computer-Aided Civil and Infrastructure Engineering 19(5), 377-392.

Verhoef, E. T. (2002a). Second-best congestion pricing in general networks. heuristic algorithms for finding second-best optimal toll levels and toll points. Transportation Research Part B 36(8), 707-729.

Verhoef, E. T. (2002b). Second-best congestion pricing in general static transportation networks with elastic demands. Regional Science and Urban Economics 32(3), 281-310.

Verhoef, E. T., P. Nijkamp, and P. Rietveld (1996). Second-best congestion pricing: the case of an untolled alternative. Journal of Urban Economics 40(3), 279-302. 
Wang, D. Z. and H. K. Lo (2010). Global optimum of the continuous network design problem with equilibrium flows. Transportation Research Part B 44(4), $482-492$.

Wardrop, J. (1952). Some theoretical aspects of road traffic research. In Proceedings of the Institute of Civil Engineers, Volume 1, pp. 325-378.

Wicaksono, D. S. and I. A. Karimi (2008). Piecewise milp under- and overestimators for global optimization of bilinear programs. AICHe Journal 54(4), 991-1008.

Yang, H. and M. G. H. Bell (1998). Models and algorithms for road network design: A review and some new developments. Transport Reviews 18(3), $257-278$.

Yang, H. and X. Zhang (2003). Optimal toll design in second-best link-based congestion pricing. Transportation Research Records (1857), 85-92.

Yildirim, M. B. (2001). Congestion Toll Pricin Models and Methods for Variale Demand Networks. Ph. D. thesis, University of Florida, Gainsville.

Yin, Y. and S. Lawphongpanich (2009). Alternative marginal-cost pricing for road networks. NETNOMICS: Economic Research and Electronic Networking 10(1), 77-83.

Zhang, X. and H. Yang (2004). The optimal cordon-based network congestion pricing problem. Transportation Research Part B 38(6), 517-537. 